TITLE:

\title{
Vibrational spectroscopy of a harmonic oscillator system nonlinearly coupled to a heat bath
}

$\operatorname{AUTHOR}(S):$

Kato, T; Tanimura, Y

\section{CITATION:}

Kato, T ... [et al]. Vibrational spectroscopy of a harmonic oscillator system nonlinearly coupled to a heat bath. JOURNAL OF CHEMICAL PHYSICS 2002, 117(13): 6221-6234

ISSUE DATE:

2002-10-01

URL:

http://hdl.handle.net/2433/49969

\section{RIGHT:}

Copyright 2002 American Institute of Physics. This article may be downloaded for personal use only. Any other use requires prior permission of the author and the American Institute of Physics. 


\title{
Vibrational spectroscopy of a harmonic oscillator system nonlinearly coupled to a heat bath
}

\author{
Tsuyoshi Kato ${ }^{\text {a) }}$ and Yoshitaka Tanimura \\ Institute for Molecular Science, Myodaiji, Okazaki, Aichi 444-8585, Japan
}

(Received 20 February 2002; accepted 9 July 2002)

\begin{abstract}
Vibrational relaxation of a harmonic oscillator nonlinearly coupled to a heat bath is investigated by the Gaussian-Markovian quantum Fokker-Planck equation approach. The system-bath interaction is assumed to be linear in the bath coordinate, but linear plus square in the system coordinate modeling the elastic and inelastic relaxation mechanisms. Interplay of the two relaxation processes induced by the linear-linear and square-linear interactions in Raman or infrared spectra is discussed for various system-bath couplings, temperatures, and correlation times for the bath fluctuations. The one-quantum coherence state created through the interaction with the pump laser pulse relaxes through different pathways in accordance with the mechanisms of the system-bath interactions. Relations between the present theory, Redfield theory, and stochastic theory are also discussed. (C) 2002 American Institute of Physics. [DOI: 10.1063/1.1503778]
\end{abstract}

\section{INTRODUCTION}

The vibrational spectra of molecules are determined by their three-dimensional structures and their vibrational force fields. An analysis of these [usually infrared (IR) and Raman] spectra can therefore provide information on the structures and on the intra- and intermolecular interactions. Over the years, normal-mode calculations have provided insight into the spectra of molecules in the condensed phases. ${ }^{1-5}$ The normal-mode frequencies are obtained by solving the secular equation that must be satisfied if the molecule is to have harmonic modes of vibration. The molecular motion can then be decomposed into normal modes, by defining new coordinates that describe the system as a collection of uncoupled harmonic oscillators.

For a molecular system in the condensed phase interactions may be divided into two categories: intramolecular and intermolecular. In such a case, instead of employing an ensemble of uncoupled oscillators, it is natural to consider a primary system corresponding to the intramolecular vibration interacting with a bath of harmonic oscillators for the intermolecular ones. This is a Brownian oscillator description of molecular vibrations. ${ }^{6-13}$ This description is especially useful to analyze vibrational spectra where the laser field only interacts with Raman or infrared active modes. One can regard such modes as the primary modes whereas the others are the bath modes. The conventional Brownian oscillator model assumes a linear-linear system-bath interaction (LL interaction) with respect to the vibrational coordinates; therefore, physically, it is equivalent to the normalmode model, and one can reduce to the normal-mode model by diagonalizing the total Hamiltonian of the Brownian oscillator model. Although the normal-mode and Brownian oscillator models seem to give reasonable explanations of vibrational spectra, in a real system, interactions between atoms or molecules are nonlinear, and the harmonic descrip-

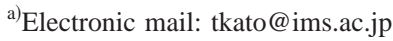

tion of the system fails. Such nonlinear interactions contribute to the energy dissipation and the phase relaxation, and may play an important role in the vibrational spectrum. The use of an explicit molecular model for this problem is computationally too expensive and it complicates the understanding of the problem.

When Okumura and Tanimura introduced the generalized Brownian oscillator model with a nonlinear systembath interaction, ${ }^{14}$ they expressed the interaction as square in the system coordinate and linear in the bath coordinate (SL interaction) and calculated a two-time correlation function of the system coordinate perturbatively. Their result was analytical, but its applicability was limited. Then, Steffen and Tanimura derived a quantum Fokker-Planck equation with the SL interaction ${ }^{15,16}$ by generalizing the quantum FokkerPlanck equation for a Gaussian-Markovian bath ${ }^{17,18}$ and calculated two-, three-, and four-time correlation functions of the Raman polarizability or the dipole moment for various coupling strengths and noise correlation times in the context of two-dimensional spectroscopy. ${ }^{6,19,20}$ It was shown that the fast modulation limit of the SL model corresponds to a situation with a homogeneous distribution of oscillators, and the slow modulation limit to an inhomogeneous distribution of oscillators; the Raman or infrared signals calculated from the three- and four-time correlation functions show the echolike feature only in the inhomogeneous case. ${ }^{16,21,22}$ In these studies, they investigated only the effects of the SL interaction assuming that the LL interactions are very small, but, in general, the LL interaction plays a role as well. In this study, we consider both the LL and SL interactions and discuss the interplay between the two.

In the next section, we present a description of the LL and SL system-bath couplings. The derivation of the Fokker-Planck equation for an oscillator system in contact with the Gaussian-Markovian noise bath is given in Appendix A. Difference between the present theory and the Redfield theory is discussed in Appendix C. In Sec. III, we dis- 
cuss third-order Raman vibrational spectroscopy. The response function formalism is also summarized to relate with the wave packet dynamics in the correlation function. After a brief explanation on the computational method in Sec. IV, the wave packet motions and the time evolutions of the coherences between the vibrational levels of a harmonic oscillator system are calculated and compared with the results from the conventional Brownian oscillator model. A selection rule that determines the vibrational relaxation pathways after the photoexcitation of the system is discussed focusing on the presence of both the LL and SL interactions. We also analyze the optical response functions and spectra by changing the physical parameters such as the coupling strength, the temperature and the correlation time of the bath fluctuations, and compare with the stochastic theory. The concluding remarks are given in Sec. V.

\section{FOKKER-PLANCK EQUATION FOR AN OSCILLATOR SYSTEM NONLINEARLY COUPLED WITH HEAT BATH OSCILLATORS}

\section{A. Hamiltonian}

Consider an optically active molecular vibrational mode in the condensed phase, which couples to the optically inactive bath degrees of freedom. The Hamiltonian of the whole system is expressed as

$$
\begin{aligned}
\hat{H}= & \frac{1}{2 M} \hat{p}^{2}+U(\hat{q})+\sum_{j} \\
& \times\left[\frac{1}{2 m_{j}} \hat{p}_{j}^{2}+\frac{1}{2} m_{j} \omega_{j}^{2}\left\{\hat{x}_{j}-\frac{F_{j}(\hat{q})}{m_{j} \omega_{j}^{2}}\right\}^{2}\right] .
\end{aligned}
$$

Here, $\hat{q}, \hat{p}, M$, and $U(\hat{q})$ denote, respectively, the coordinate (displacement from the potential minimum), momentum, reduced mass, and the potential of the optically active oscillator, which is called the system in the following discussion. The bath degrees of freedom are treated as an ensemble of harmonic oscillators, and the coordinate, momentum, mass, and frequency of the $j$ th bath oscillator are given by $\hat{q}_{j}, \hat{p}_{j}$, $m_{j}$, and $\omega_{j}$, respectively. The system-bath interaction is given by

$$
\hat{H}_{\mathrm{SB}}=-\sum_{j} \hat{x}_{j} F_{j}(\hat{q})
$$

where only the linear dependence on the bath coordinates is taken into account. For the system coordinate $\hat{q}$ we include terms up to second order,

$$
F_{j}(\hat{q})=\frac{g_{j}}{2}\left(2 C_{1} \hat{q}+C_{2} \hat{q}^{2}\right) \equiv \frac{g_{j}}{2} V(\hat{q}),
$$

where $g_{j}$ denotes the coupling strength to the $j$ th bath mode. The interaction described by Eqs. (2.2) and (2.3) are the first few terms of the general system-bath interaction Hamiltonian, $\hat{H}_{S B}^{\prime}=\Sigma_{j}\left(a_{j} \hat{x}_{j}+\cdots\right)\left(b \hat{q}+b^{\prime} \hat{q}^{2}+\cdots\right){ }^{23}$ Constants $C_{1} \geqslant 0$ and $C_{2}$ are introduced to specify the relative importance of the couplings via the $\hat{q}$ or $\hat{q}^{2}$ term, respectively. For the sake of this discussion only the ration $C_{1} / C_{2}$ is necessary, but it is convenient to leave them in the present formu-

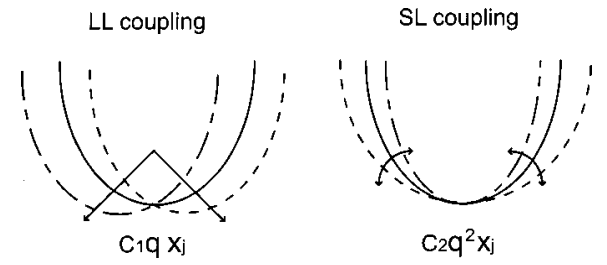

FIG. 1. Schematic illustrations of the effects of the system-bath coupling on a relevant harmonic potential system. The LL coupling and the SL coupling induce the displacement and the fluctuation of the relevant potential, respectively, and are responsible for $T_{1}$-type and $T_{2}$-type vibrational relaxation processes in the weak coupling limit.

lation. We refer to the bilinear coordinate coupling term proportional $C_{1}$ as the linear-linear (LL) coupling term, and the term proportional to $C_{2}$ as the square-linear (SL) coupling term. The dimensionless constant $C_{2}$ will be set to either 1 or 0, i.e., the system-bath interaction either includes the SL coupling or it does not. The LL model has been widely used in studying the effects of dissipative processes in quantum dynamics $^{7-10}$ such as tunneling ${ }^{9,18}$ and curve-crossing problems. $^{24,25}$ In the field of spectroscopy such coupling mechanisms have been used to describe energy dissipation from the vibrational system to the heat bath modes, which is called population decay or $T_{1}$-type relaxation. ${ }^{6}$ In addition to the energy dissipation due to the LL coupling, we expect that system potential fluctuations will arise due to the presence of the SL coupling, which is related to pure dephasing or $T_{2}$-type relaxation. ${ }^{26-28}$ These notions of the relaxation phenomena are made for a harmonic oscillator system denoted by the creation and annihilation operators, $\hat{a}^{\dagger}$ and $\hat{a}$, respectively, with a weak system-bath coupling. This is equivalent to the harmonic system with the coordinate operator $\hat{q}$ $=\sqrt{\hbar /\left(2 M \omega_{0}\right)}\left(\hat{a}+\hat{a}^{\dagger}\right)$, where $\omega_{0}$ is the fundamental frequency. It can be understood that the LL coupling $(\propto \hat{a}$ $+\hat{a}^{\dagger}$ ) leads to one-quantum transitions of the system, while the SL coupling $\left(\propto 1+2 \hat{a}^{\dagger} \hat{a}+\hat{a}^{2}+\hat{a}^{\dagger 2}\right)$ implies elastic interaction $\left(1+2 \hat{a}^{\dagger} \hat{a}\right)$ as well as two-quantum transitions $\left(\hat{a}^{2}\right.$ $+\hat{a}^{\dagger 2}$ ) as shown in Fig. 1 [see Eqs. (2.16)].

\section{B. Specification of the bath modes}

To derive a Fokker-Planck equation which describes the system dynamics in the presence of both the LL and SL couplings with the heat bath, one has to define a spectral density $J(\omega)$. We employ the spectral density relevant to the SL coupling

$$
J(\omega)=\sum_{j} \frac{g_{j}^{2}}{8 m_{j} \omega_{j}} \delta\left(\omega-\omega_{j}\right) \Rightarrow \frac{M \zeta}{\pi} \frac{\omega \gamma^{2}}{\omega^{2}+\gamma^{2}},
$$

where an infinite number of bath modes is assumed and the summation of Dirac delta functions is replaced by a continuous function of $\omega$. The constant $\gamma$ represents the width of the spectral distribution of the bath modes and is related to the correlation time of the noise induced by the bath

$$
\tau_{c} \equiv 1 / \gamma
$$


To see this relationship, we define the interaction coordinate of the bath modes as

$$
\hat{X} \equiv \sum_{j} \frac{g_{j}}{2} \hat{x}_{j}
$$

Within the high temperature approximation $\beta \hbar \gamma \ll 1 \quad(\beta$ $=1 / k_{B} T$, with $k_{B}$ and $T$ being the Boltzmann constant and temperature, respectively), the symmetrized correlation function of the interaction coordinate takes a single exponential decay form ${ }^{29}$

$$
S(t) \equiv \frac{1}{2}\langle\hat{X}(t) \hat{X}(0)+\hat{X}(0) \hat{X}(t)\rangle_{B}=\frac{M \zeta \gamma}{\beta} e^{-\gamma|t|},
$$

where $\hat{X}(t)$ is the Heisenberg operator of $\hat{X}$ and $\langle\cdots\rangle_{B}$ means taking the thermal average with respect to the bath degrees of freedom. Equation (2.7) states that the bath oscillators disturb the system with Gaussian-Markovian noise. The Gaussian nature is apparent since the harmonic oscillator bath is completely characterized by the second-order cummulant. $^{30}$

\section{Fokker-Planck equation for a Gaussian-Markovian noise bath}

In this subsection, we present the quantum FokkerPlanck equation with the LL and SL interactions for the Gaussian-Markovian noise bath. This equation is valid for colored Gaussian-Markovian noise from weak to strong system-bath coupling under the high-temperature condition $\hbar \gamma \ll k_{B} T$. The procedure to derive the equation of motion is described in detail in Refs. 16 and 17, where a prescription based on the influence functional formalism is utilized. ${ }^{31} \mathrm{We}$ summarize it for the reduced density matrix $\rho\left(q, q^{\prime}, t\right)$, including both the LL and SL interactions, in Appendix A. Here, we write it in the Wigner representation. ${ }^{32}$ The Wigner distribution function is the quantum analog of the classical distribution function in phase space, and is defined as

$$
W(p, q, t) \equiv \frac{1}{2 \pi \hbar} \int_{-\infty}^{\infty} d r e^{i p r / \hbar} \rho\left(q-\frac{r}{2}, q+\frac{r}{2}, t\right) .
$$

The Wigner distribution function is a real function in contrast to the complex density matrix. Wigner transformation of the equations of motion for the reduced density matrix derived in Appendix A yields the quantum Fokker-Planck equation for the Gaussian-Markovian noise bath

$$
\frac{\partial}{\partial t} W_{0}(p, q, t)=-\mathcal{L}_{s} W_{0}(p, q, t)-\Phi_{W}(p, q) W_{1}(p, q, t),
$$

$$
\begin{aligned}
\frac{\partial}{\partial t} W_{1}(p, q, t)= & -\left\{\mathcal{L}_{s}+\gamma\right\} W_{1}(p, q, t) \\
& -\Phi_{W}(p, q) W_{2}(p, q, t) \\
& +\Theta_{W}(p, q) W_{0}(p, q, t),
\end{aligned}
$$

$$
\begin{aligned}
\frac{\partial}{\partial t} W_{n}(p, q, t)= & -\left\{\mathcal{L}_{s}+n \gamma\right\} W_{n}(p, q, t) \\
& -\Phi_{W}(p, q) W_{n+1}(p, q, t) \\
& +n \Theta_{W}(p, q) W_{n-1}(p, q, t),
\end{aligned}
$$

$$
\begin{aligned}
\frac{\partial}{\partial t} W_{N}(p, q, t)= & -\left\{\mathcal{L}_{s}+N \gamma\right\} W_{N}(p, q, t) \\
& +\Gamma_{W}(p, q) W_{N}(p, q, t) \\
& +N \Theta_{W}(p, q) W_{N-1}(p, q, t) .
\end{aligned}
$$

In Eqs. (2.9), only $W_{0}(p, q, t) \equiv W(p, q, t)$ has physical meaning and the others, $W_{n}(p, q, t)(1 \leqslant n \leqslant N)$, are auxiliary functions being introduced to avoid the explicit treatment of the inherent memory effects during the time evolution of the reduced density matrix [see Eqs. (A2) and (A4)]. By the exponential decay assumption of the bath correlation function [Eq. (2.7)], the time nonlocality in the equation of motion is taken into account as the hierarchical structure of the above expression; the time evolution is described by a set of coupled Markovian-type (first-order derivative in time) equations, which allows us to implement numerical calculations using well-established methods. ${ }^{33}$ The depth of the hierarchy $N$ is chosen according to the time scale of the correlation time $1 / \gamma$ and the strength of the system-bath interactions [see Sec. IV].

Some details of transformations from the coordinate representation to the Wigner representation are listed in Appendix B. Here, we give the explicit expressions of operators in Eqs. (2.9). The action of the system Liouvillian on the Wigner function is defined by

$$
\begin{aligned}
-\mathcal{L}_{s} W_{n}(p, q, t) \equiv & -\frac{p}{M} \frac{\partial}{\partial q} W_{n}(p, q, t) \\
& -\frac{1}{\hbar} \int_{-\infty}^{\infty} \frac{d p^{\prime}}{2 \pi \hbar} V\left(p-p^{\prime}, q\right) W_{n}\left(p^{\prime}, q, t\right)
\end{aligned}
$$

with the potential kernel

$$
V(p, q) \equiv 2 \int_{0}^{\infty} d r \sin (p r / \hbar)\left\{U\left(q+\frac{r}{2}\right)-U\left(q-\frac{r}{2}\right)\right\} .
$$

The relaxation operators induced by the system-bath interactions are given by

$$
\begin{aligned}
& \Phi_{W}(p, q)=-2\left(C_{1}+C_{2} q\right) \frac{\partial}{\partial p}, \\
& \Theta_{W}(p, q)=2 \zeta \gamma\left\{\left(C_{1}+C_{2} q\right)\left(p+\frac{M}{\beta} \frac{\partial}{\partial p}\right)+C_{2} \frac{\hbar^{2}}{4} \frac{\partial^{2}}{\partial p \partial q}\right\},
\end{aligned}
$$




$$
\begin{aligned}
\Gamma_{W}(p, q)= & 4 \zeta\left(C_{1}+C_{2} q\right)^{2} \frac{\partial}{\partial p}\left(p+\frac{M}{\beta} \frac{\partial}{\partial p}\right) \\
& +\hbar^{2} \zeta C_{2}\left(C_{1}+C_{2} q\right) \frac{\partial^{3}}{\partial p^{2} \partial q} .
\end{aligned}
$$

We shall represent the SL coupling between the system and the heat bath by a set of parameters $\zeta_{\mathrm{SL}}$ and $\gamma$. Here, $\zeta_{\mathrm{SL}}$ $\equiv \zeta C_{2}$ denotes the SL coupling strength and has the dimension of $m^{-2} s^{-1}$. According to Eqs. (2.3) and (2.4), the LL coupling strength $\zeta_{\mathrm{LL}}$ is also defined by

$$
\zeta_{\mathrm{LL}}=4\left[C_{1}\right]^{2} \zeta
$$

with the dimension $s^{-1}$. If we put $C_{1} \equiv 0$, i.e., the SL model, the above equations, Eqs. (2.9)-(2.12), reduce to that derived in Refs. 15 and 16 as should be, while $C_{2} \equiv 0$ leads to the conventional Brownian oscillator model. ${ }^{17,18}$ Thus, the present Fokker-Planck equation unifies the SL coupling model considered in Refs. 15 and 16 and the Brownian oscillator model.

The Gaussian-white noise bath represented by the Ohmic spectral density

$$
J(\omega)=M \zeta_{\mathrm{SL}} \omega / \pi,
$$

is the limit of the Gaussian-Markovian bath with a vanishingly short correlation time compared to the characteristic time scale of the system, e.g., $\quad \gamma \rightarrow \infty$ and $S(t)$ $\rightarrow 2 M \zeta_{\mathrm{SL}} \delta(t) / \beta$. A Fokker-Planck equation for the Gaussian-white noise bath can be derived by following the procedure described in Appendix A, but is quickly obtained by formally putting $N=0$ in Eq. (2.9) as

$$
\frac{\partial}{\partial t} W(p, q, t)=-\mathcal{L}_{s} W(p, q, t)+\Gamma_{W}(p, q) W(p, q, t),
$$

in which the time evolution of the system is described by a Markov-type differential equation. Physically, Eq. (2.14) is valid when $\gamma \gg \omega_{c}$, where $\omega_{c}$ is the characteristic frequency of the system such as the frequency of the harmonic potential, thus, the high-temperature condition now implies $\beta \hbar \omega_{c} \ll 1 .^{10,17}$ Equation (2.14) is also derived by a secondorder perturbation theory and the six different approaches have been carefully compared for the LL coupling model. ${ }^{34}$ We see that the numerical evaluation of the GaussianMarkovian equations of motion [Eq. (2.9)] is more time consuming than that of the Gaussian-white equation of motion, however, the former can be applied with more moderate restriction on the bath temperatures. ${ }^{17}$

For comparison, we present some of Redfield tensor elements ${ }^{28,35-37}$ for the present model (cf. Appendix C). The Redfield theory has been utilized to describe quantum dissipative dynamics by the reduced density matrix in the energylevel representation, ${ }^{36}$ where the system-bath interactions are treated with second-order perturbation theory. There are two important tensor elements within the rotating wave approximation (RWA) (or secular approximation), ${ }^{28,37,34}$ i.e., $R_{n, n ; m, m}$ and $\operatorname{Re}\left[R_{n, m ; n, m}\right]$ describing the transition rate from $m$ th vibrational level of the system $|m\rangle$ to the $n$th level and the dephasing rate between these levels, respectively. These are given by

$$
\begin{aligned}
R_{n, n ; m, m}= & \frac{2 \pi}{\hbar}|\langle n|V(\hat{q})| m\rangle|^{2} \int_{0}^{\infty} d \omega J(\omega)\left[N(\omega) \delta\left(\omega-\omega_{n m}\right)\right. \\
& \left.+\{N(\omega)+1\} \delta\left(\omega+\omega_{n m}\right)\right](n \neq m),
\end{aligned}
$$

and

$$
\begin{aligned}
\operatorname{Re}\left[R_{n, m ; n, m}\right]= & -\frac{1}{2}\left(\kappa_{n}+\kappa_{m}\right)-\frac{2}{\hbar}\{\langle n|V(\hat{q})| n\rangle \\
& -\langle m|V(\hat{q})| m\rangle\}^{2} \int_{0}^{\infty} d \tau \int_{0}^{\infty} d \omega J(\omega) \\
& \times\left\{N(\omega)+\frac{1}{2}\right\} \cos (\omega \tau)(n \neq m), \quad
\end{aligned}
$$

where the notation $\kappa_{m} \equiv \sum_{r \neq m} R_{r, r ; m, m}$ is used for the population decay rate out of the $m$ th vibrational level and $\omega_{n m}$ $=\omega_{n}-\omega_{m}$ for the frequency difference between the relevant levels. $N(\omega)=1 /\left(e^{\beta \hbar \omega}-1\right)$ is the thermal occupation number of the harmonic oscillator of the frequency $\omega$, and $V(\hat{q})$ is defined in Eq. (2.3). The transition rate satisfies the detailed balance condition, e.g., $R_{n-1, n-1 ; n, n}$ $=e^{\beta \hbar \omega_{n, n-1}} R_{n, n ; n-1, n-1}$. For a harmonic oscillator system with fundamental frequency $\omega_{0}$ and the Gaussian-white bath represented by Eq. (2.14), the above tensor elements reduce to

$$
\begin{aligned}
R_{n, n ; m, m}= & m \zeta_{\mathrm{LL}}\left\{N\left(\omega_{0}\right)+1\right\} \delta_{n, m-1}+(m+1) \zeta_{\mathrm{LL}} \\
& \times N\left(\omega_{0}\right) \delta_{n, m+1}+m(m-1) \hbar \zeta_{\mathrm{SL}}\left\{N\left(2 \omega_{0}\right)+1\right\} / \\
& \left(M \omega_{0}\right) \delta_{n, m-2}+(m+2)(m+1) \hbar \zeta_{\mathrm{SL}} N\left(2 \omega_{0}\right) / \\
& \left(M \omega_{0}\right) \delta_{n, m+2},
\end{aligned}
$$

and

$$
\operatorname{Re}\left[R_{n, m ; n, m}\right]=-\frac{1}{2}\left(\kappa_{n}+\kappa_{m}\right)-\frac{\zeta_{\mathrm{SL}}}{M \beta \omega_{0}^{2}}(n-m)^{2},
$$

respectively. The second term of Eq. (2.16b) represents the pure dephasing rate constant and its magnitude is proportional to the square of the difference of the quantum numbers of the relevant vibrational levels..$^{20,26,27,38-41}$ Because of the RWA, expressions in Eqs. (2.16) are valid regardless of the statistics of the bath modes, i.e., the bath modes that contribute to the LL coupling and those to the SL coupling can be independent from each other. We can see, on the other hand, the interferences between the effect from the LL coupling and that from the SL coupling as the cross terms being proportional to $C_{1} C_{2}$ in the relaxation operator of Eq. (2.11c). These interferences will vanish when the spectral overlap between the bath modes that participate in the LL coupling and those that participate in the SL coupling are absent. Effects of this interference might be described by the Redfield tensor elements beyond the RWA.

To conclude this section, we mention an alternative form of a Fokker-Planck equation investigated by Yan, ${ }^{42,43}$ with which one can handle a non-Markovian heat bath within two 
different partial resummation schemes. In the Markovian bath limit, Yan's two prescriptions result in a Fokker-Planck equation consisting of two coupled differential equations, ${ }^{44}$ where the number of coupled equations $(N=2)$ is independent of the bath correlation time. It consists of a single differential equation for the white bath case and agrees with our Fokker-Planck equation in Eq. (2.14) applied to the LL coupling model. ${ }^{10,17}$ Quite recently, a fourth-order perturbation theory was applied to derive a quantum master equation for the Markovian heat bath model, and the relation between the path integral formalism and the perturbation treatment was discussed. ${ }^{45}$

\section{OPTICAL RESPONSE FUNCTION FOR VIBRATIONAL SPECTROSCOPY}

In this section, we present an expression for the nonresonant third-order Raman spectroscopy on the oscillator system, which is a correlation function of the system coordinate, to demonstrate the effects of the vibrational relaxation for different system-bath coupling mechanisms. In this spectroscopy, molecular vibrations in the electronic ground state are coherently excited by the pumping laser pulse $E(t)$ via an interaction through the polarizability $\alpha(\hat{q})$ followed by the probe laser pulse $E_{T}(t)$, which induces the Raman polarization to be detected. The time separation between the pump and the probe pulse, denoted by $T_{1}$, is controlled in the time-resolved experiment. ${ }^{6}$ The Hamiltonian including the pumping laser field is

$$
\hat{H}_{\text {Raman }}=\hat{H}-\alpha(\hat{q}) E(t)^{2} .
$$

Note that since the Hamiltonian for the resonant infrared (IR) spectroscopy is given by $\hat{H}_{I R}=\hat{H}-\mu(\hat{q}) E(t)$ with the dipole operator $\mu(\hat{q})$, one can treat the linear IR response by formally replacing the polarizability operator $\alpha(\hat{q})$ by the dipole operator $\mu(\hat{q})$ in Eqs. (3.4) and (3.5) below. In the following, we will discuss Raman spectroscopy, but the same results can be applied to IR spectroscopy by appropriately replacing the physical quantities involved.

For simplicity, we assume that the laser fields are impulsive, and defined by,

$$
E(t)=\mathcal{E} \delta(t) \text { and } E_{T}(t)=\mathcal{E}_{T} \delta\left(t-T_{1}\right),
$$

where $\mathcal{E}$ denotes the pulse area. The Raman polarization is calculated by using perturbation theory with respect to the system-laser field interactions and is expressed as 6,19

$$
P(t)=\mathcal{E}_{T} \mathcal{E}^{2} \delta\left(t-T_{1}\right) R\left(T_{1}\right),
$$

with the Raman response function

$$
R\left(T_{1}\right) \equiv \frac{i}{\hbar}\left\langle\left[\hat{\alpha}\left(T_{1}\right), \hat{\alpha}(0)\right]\right\rangle=\left\langle\alpha(\hat{q}) e^{-i \mathcal{L} T_{1} / \hbar} \frac{i}{\hbar} \alpha^{\times}(\hat{q})\right\rangle,
$$

where $\hat{\alpha}(t)=e^{i \hat{H} t / \hbar} \alpha(\hat{q}) e^{-i \hat{H} t / \hbar}$ is the polarizability operator in the Heisenberg representation, and $\langle\cdots\rangle=\operatorname{Tr}\left\{\cdots \hat{\rho}_{\text {eq }}\right\}$, in which $\hat{\rho}_{\mathrm{eq}} \equiv e^{-\hat{\beta} \hat{H}} / \operatorname{Tr}\left\{e^{-\beta \hat{H}}\right\}$ means taking the thermal average with respect to the system and the bath degrees of freedom. We use the notation $A^{\times} \cdots \equiv[\hat{A}, \cdots]$ for the Liouville space operator, and $\mathcal{L} \cdots \equiv[\hat{H}, \cdots]$ for the Liouvillian in the rightmost expression in Eq. (3.4). We can make it further suitable for numerical calculations by the use of our FokkerPlanck equation. For example, in the Gaussian-white noise bath [Eq. (2.14)], it is given by ${ }^{46}$

$$
R\left(T_{1}\right)=\operatorname{Tr}\left\{\alpha_{W}^{\circ} e^{-\left(L_{W}^{0}-\Gamma_{W}\right) T_{1}} \alpha_{W} W^{\mathrm{eq}}\right\},
$$

where $W^{\mathrm{eq}}(p, q)$ stands for the thermal equilibrium Wigner distribution function of the system (see the next section), and Tr means the integration over $p$ and $q$ variables. The actions of the Wigner operators corresponding to the commutator $\alpha_{W}$ and the anticommutator $\alpha_{W}{ }^{\circ}$ on the distribution function are given in Appendix B [Eqs. (B3) and (B4)]. For the Gaussian-Markovian noise bath, the Wigner distribution function and time-evolution operators are cast into the hierarchical form. The operator $\alpha_{W}$ and $\alpha_{W}{ }^{\circ}$ operate throughout the hierarchical elements $W_{n}(p, q, t)(0 \leqslant n \leqslant N)$. After the time evolution and operation of $\alpha_{W}{ }^{\circ}$, the zeroth order element $W_{0}(p, q, t)$ is used to calculate the signal. ${ }^{15}$

\section{NUMERICAL RESULTS AND DISCUSSION}

Here, we note some details of the numerical calculations. In the following calculations, we employed the dimensionless momentum and coordinate defined as $P \equiv p / \sqrt{\hbar M \omega_{0}}$ and $Q \equiv q \sqrt{M \omega_{0} / \hbar}$, respectively. The SL coupling strength $\zeta_{\mathrm{SL}}$ is determined by the dimensionless coupling strength $\zeta_{\mathrm{SL}}^{\prime} \equiv \hbar \zeta_{\mathrm{SL}} /\left(M \omega_{0}^{2}\right)$. The magnitude of $C_{1}$ is determined from Eq. (2.12), which accounts for the influences of the LL coupling even when the SL coupling is absent, i.e., $C_{2} \equiv 0$ in Eq. (2.9). We assume the harmonic potential, $U(q)$ $=M \omega_{0}^{2} q^{2} / 2$, for the system oscillator where the fundamental frequency is set to be $\hbar \omega_{0}=38.7 \mathrm{~cm}^{-1}\left(T_{v}=861 \mathrm{fs}\right)$ being the same value as our previous papers. ${ }^{15,16}$ Although Eqs. (2.9) can be applied to high-frequency vibrations $(\sim 1000$ $\mathrm{cm}^{-1}$ ), we limit our calculations for a low-frequency mode, since in addition to the SL interaction, one needs to take into account the anharmonicity of the potential, which should be studied as a separated work. The second term on the righthand side of Eq. (2.10a) reduces to $M \omega_{0}^{2} q \partial W_{n}(p, q, t) / \partial p$ for a harmonic potential. The polarizability operator is assumed to be $\alpha(q)=\alpha_{1} q$. Then, the expression of the Wigner operator $\alpha_{W}$ can be cast into a differential form as $-\alpha_{1} \partial / \partial p$.

The thermal equilibrium distributions $W_{n}^{e q}(p, q)(0 \leqslant n$ $\leqslant N)$ required as the initial condition are obtained by integrating Eq. (2.9) or (2.14) from time $t=-t_{i}<0$ to $t=0$ with trial initial conditions

$$
W_{0}\left(p, q,-t_{i}\right)=\mathcal{N} e^{-\beta\left(p^{2} /(2 M)+U(q)\right)},
$$

and

$$
W_{n}\left(p, q,-t_{i}\right)=0(n=1, \ldots, N),
$$

where $\mathcal{N}$ is the normalization constant. During the time evolution for the interval $t_{i} \geqslant 1 / \gamma$, auxiliary functions $W_{n}(p, q, 0)(1 \leqslant n \leqslant N)$ become nonzero and the wave packets become stable. The depth of the hierarchy $N$ should fulfill $N \gamma>4 \omega_{0}$ and $N \gamma>4 \zeta_{\text {SL }}$ from the numerical simulations. ${ }^{16}$ We notice that the distribution of Eq. (4.1a) is not an equi- 
librium state except for the case of the Gaussian-white noise bath $\left(\gamma \gg \omega_{c}\right)$, since it neglects initial correlation between the system and bath. ${ }^{8,47}$ therefore we need to generate the thermal equilibrium states by numerically integrating Eqs. (2.9) as described above. ${ }^{17,48}$

The numerical integrations are performed by using a second-order Runge-Kutta method applied to a finite difference expression of Eq. (2.9) or (2.14) ${ }^{49}$ on a discrete mesh in the phase space. The time steps for the finite difference expression for $\partial W_{n} / \partial t$ were between 0.004 and $0.05 \mathrm{fs}$. The mesh size was varied between $80 \times 300$ and $100 \times 500$ for the mesh range $-11 \leqslant P \leqslant 11$ and $-15 \leqslant Q \leqslant 15$, and $-15 \leqslant P$ $\leqslant 15$ and $-15 \leqslant Q \leqslant 15$. The accuracy of the calculations was checked by changing the hierarchy depth $N$, the mesh size, and the time step.

\section{A. Wave packet motions}

In this subsection, we examine the effects of the SL coupling under the presence of the LL coupling by plotting the wave packet motions initiated by the interaction with the impulsive pump pulse. The reduced density matrix responsible for the Raman response function is defined from Eq. (3.4) by

$$
\rho\left(T_{1}\right)=\left\langle e^{-i \mathcal{L} T_{1} / \hbar} \frac{i}{\hbar} \alpha^{\times}(\hat{q}) \hat{\rho}_{e q}\right\rangle .
$$

Accordingly, we define the wave packet in the phase space as

$$
W_{0}\left(T_{1}\right)=e^{-\left(L_{W}^{0}-\Gamma_{W}\right) T_{1}} \alpha_{W} W_{0}^{\mathrm{eq}} .
$$

The wave packet motions of $W_{0}\left(p, q, T_{1}\right)$ are plotted in Figs. 2-4 for the LL coupling, the SL coupling, and the LL plus SL coupling models, respectively. Here, we assumed the Gaussian-white bath with a temperature of $300 \mathrm{~K}$. The coupling strengths are $\zeta_{\mathrm{LL}}=10 \mathrm{~cm}^{-1}\left[\zeta_{\mathrm{LL}} /\left(\hbar \omega_{0}\right)=0.26\right]$ and $\zeta_{\mathrm{SL}}^{\prime}=0.1$. In each figure, we also plot the thermal equilibrium wave packet in the top-left panel which is obtained by integrating the Gaussian-white Fokker-Planck equation for 5 ps. We compared the calculated results with the classical counterpart obtained from Eq. (4.1a) and found that the difference is very small. After the interaction expressed by $\alpha_{W}=-\alpha_{1} \partial / \partial p$, which results in a node on the wave packet along $P=0$ axis, the wave packet begins a clockwise rotation around the origin in the phase space and shrinks its shape because of the relaxation.

To illustrate the movements, here we also present the analytical expression for the wave packet in the LL Brownian model with Gaussian-white noise [Eq. (2.13) with Eq. (2.12)]. The Wigner function after the pump excitation is 6,12

$$
\begin{aligned}
W_{0}\left(p, q, T_{1}\right)= & -\frac{2 \alpha_{1}}{\pi} \sqrt{\frac{1}{\left\langle p^{2}\right\rangle\left\langle q^{2}\right\rangle}}\left[\frac{D\left(T_{1}\right)}{\left\langle q^{2}\right\rangle} q+\frac{M \dot{D}\left(T_{1}\right)}{\left\langle p^{2}\right\rangle} p\right] \\
& \times \exp \left\{-\frac{q^{2}}{2\left\langle q^{2}\right\rangle}-\frac{p^{2}}{2\left\langle p^{2}\right\rangle}\right\},
\end{aligned}
$$

where

$$
D(t)=-\frac{\hbar}{2 M \Omega_{0}} e^{-\zeta_{\mathrm{LL}} t / 2} \sin \left(\Omega_{0} t\right),
$$

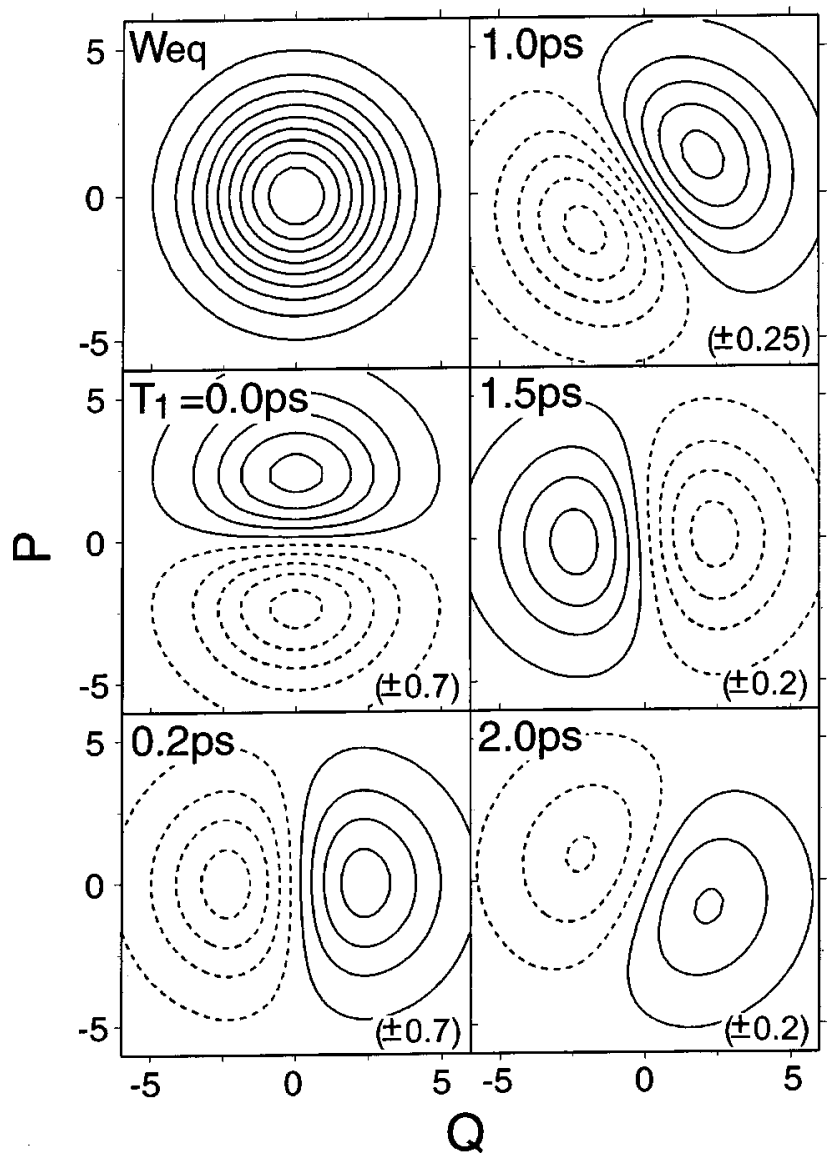

FIG. 2. The wave packet motion in the phase space for the LL coupling with the Gaussian-white bath at temperature $300 \mathrm{~K}$. Shown in the top-left panel is the equilibrium wave packet. Indicated in each panel are the evolution time $T_{1}$ and the relative height of the contour plot. The solid (broken) contours represents positive (negative values). The LL coupling strength is $\zeta_{\mathrm{LL}}=10$ $\mathrm{cm}^{-1}$.

and the dot implies the time derivative. The renormalized frequency is redshifted from $\omega_{0}$ as $\Omega_{0}=\sqrt{\omega_{0}-\zeta_{\mathrm{LL}}^{2} / 4}$, and variances $\left\langle q^{2}\right\rangle$ and $\left\langle p^{2}\right\rangle$ are given by $\hbar \operatorname{coth}\left(\beta \hbar \omega_{0}\right) /\left(2 M \omega_{0}\right)$ and $M \hbar \omega_{0} \operatorname{coth}\left(\beta \hbar \omega_{0}\right) / 2$, respectively. To derive Eq. (4.4a), we assumed the initial equilibrium distribution, then modified it by the impulsive pump pulse with a linear polarizability $(\propto-\hat{q})$ at $T_{1}=0$. The expectation values of the coordinate and the momentum at time $T_{1}$ are proportional to the prefactor of $q$ and $p$ in Eq. (4.4a), respectively.

In the LL coupling case [Fig. 2], the wave packet rotates and shrinks preserving its shape as is described by Eq. (4.4a). The underlying relaxation process is population decay limited relaxation, i.e., there is only $T_{1}$-type population transfer and the $T_{2}$-type coherence decay is due to the population decay process. ${ }^{27}$ We notice that the relaxation is governed by the momentum dependent relaxation operators of Eqs. (2.11) with $C_{2} \equiv 0$ in the LL coupling model. On the other hand, the SL coupling and the LL plus SL coupling mechanisms result in more complex wave packet motions than that for the LL coupling case as shown in Figs. 3 and 4. The relaxation operators are both coordinate- and momentum-dependent in 


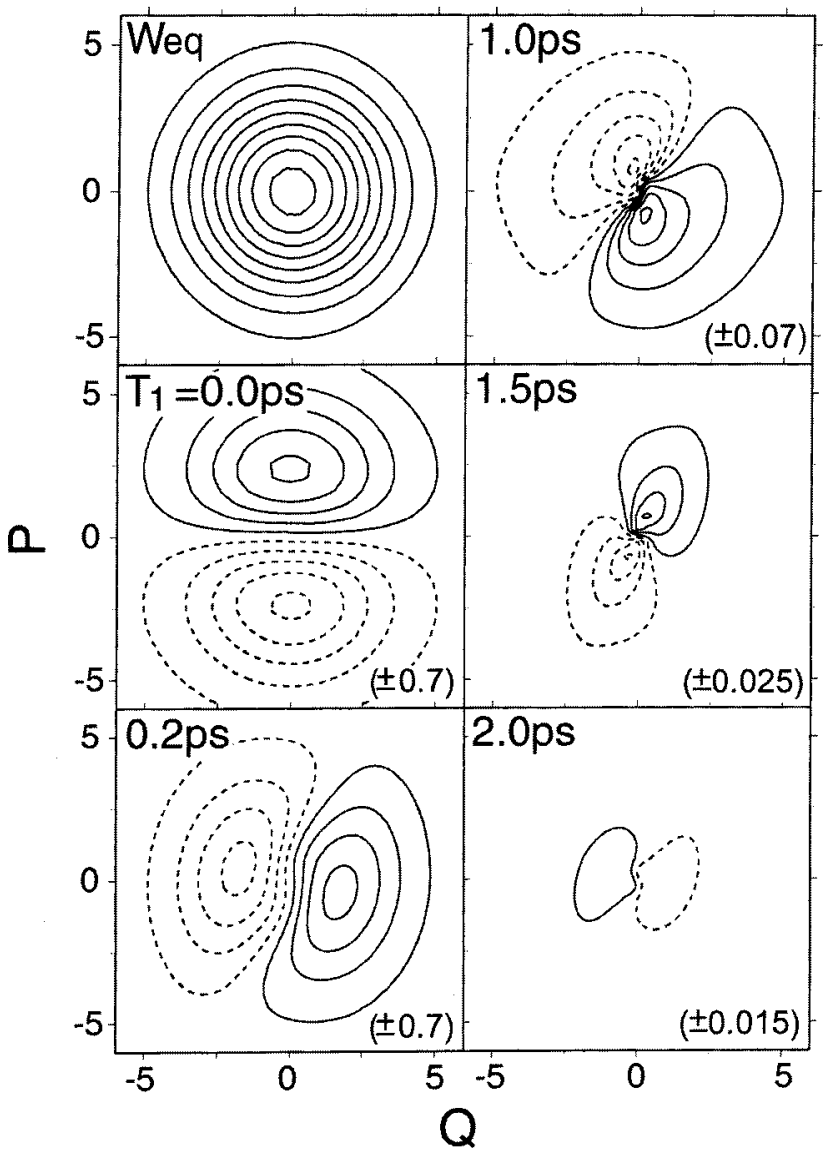

FIG. 3. The wave packet motion in the phase space for the SL coupling with the Gaussian-white bath at temperature $300 \mathrm{~K}$. The meaning of the lines and the depicted numbers are the same as in Fig. 2. The SL coupling strength is $\zeta_{\mathrm{SL}}^{\prime}=0.1$.

these cases, suggesting that the relaxation is level-dependent as was indicated by Eq. (2.16b) within the Redfield theory. The rate of shrinkage for the wave packet becomes larger than in the LL coupling model because of the pure dephasing contribution from the SL coupling [see Eq. (4.8)]. The outer part of the wave packet seems to decay faster than in the LL coupling case for the early time period $\left[T_{1} \leqslant 1.0 \mathrm{ps}\right]$, since the relaxation operators stem from the SL coupling have extra coordinate dependence than the LL coupling in Eqs. (2.11). We note that the wave packet has inversion symmetry in the SL coupling case [Fig. 3] and the LL coupling case [Fig. 2], but not in the LL plus SL coupling case [Fig. 4]. These are due to the symmetry of the system-bath interaction term [Eqs. (2.2) and (2.3)], in which the LL coupling term preserves its sign under the coordinate transformation such as $x_{j} \rightarrow-x_{j}$, whereas the SL coupling term changes its sign, but the LL plus SL coupling term possesses no such symmetry. This is regarded as the "anisotropy of the medium," through our model Hamiltonian [Eq. (2.1)] describes one-dimensional motion of the system.

To see these differences in the wave packet motions quantitatively, we introduce projection operators $\mathcal{P}_{n m}$ defined by the operation on the distribution function

$\mathcal{P}_{n m} W_{0}(t)=\int_{-\infty}^{\infty} d r e^{i p r / \hbar} \varphi_{n}^{*}(q+r / 2) \varphi_{m}(q-r / 2) W_{0}(p, q, t)$

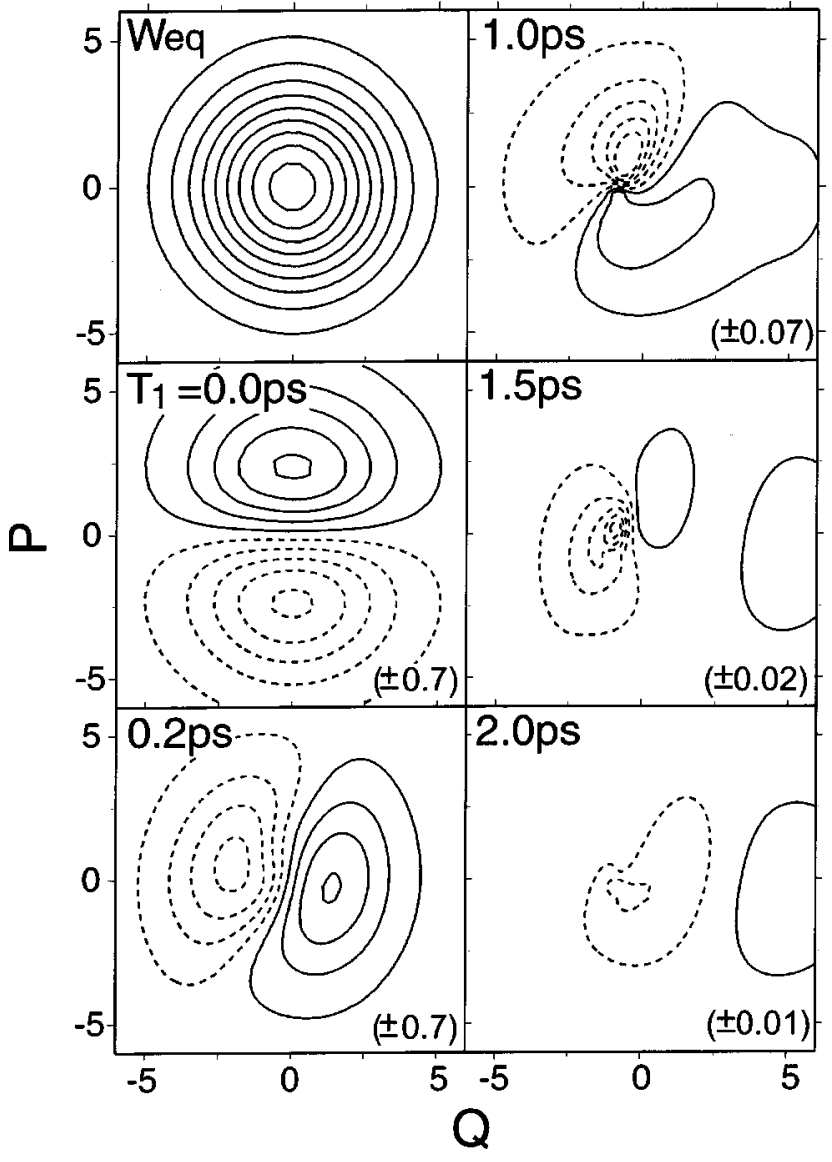

FIG. 4. The wave packet motion in the phase space for the LL plus SL coupling with the Gaussian-white bath at temperature $300 \mathrm{~K}$. The meaning of the lines and the depicted numbers are the same as in Fig. 2. The coupling parameters are $\zeta_{\mathrm{LL}}=10 \mathrm{~cm}^{-1}$ and $\zeta_{\mathrm{SL}}^{\prime}=0.1$.

for integer $n$ and $m(\geqslant 0)$, where $\varphi_{n}(q)$ is the $n$th eigenfunction of the relevant harmonic oscillator. Integration of Eq. (4.5) over $p$ and $q$ variables results in the matrix element $C_{n, m}(t) \equiv\langle n|\hat{\rho}(t)| m\rangle$ in energy-level representation. Within the Brownian harmonic oscillator model, we obtain from Eq. (4.4a)

$$
\begin{aligned}
\operatorname{Re}\left[C_{n+1, n}(t)\right]= & -\alpha_{1}^{2} \sqrt{\frac{1}{2 M \hbar \omega_{0}}}\left\{\sqrt{n+1} e^{-n \beta \hbar \omega_{0}}\right. \\
& \left.\times\left(1+e^{-\beta \hbar \omega_{0}}\right)\right\} \frac{D(t)}{\left\langle q^{2}\right\rangle},
\end{aligned}
$$

and

$$
\begin{aligned}
\operatorname{Im}\left[C_{n+1, n}(t)\right]= & -\alpha_{1}^{2} \sqrt{\frac{1}{2 M \hbar \omega_{0}}} \\
& \times\left\{\sqrt{n+1} e^{-n \beta \hbar \omega_{0}}\left(1-e^{-\beta \hbar \omega_{0}}\right)\right\} \frac{2 M \dot{D}(t)}{\hbar},
\end{aligned}
$$

where only the coherences between the adjacent vibrational levels are created.

In Fig. 5, we plot the normalized coherence as a function of time for (a) the LL coupling case: (b) the SL coupling 


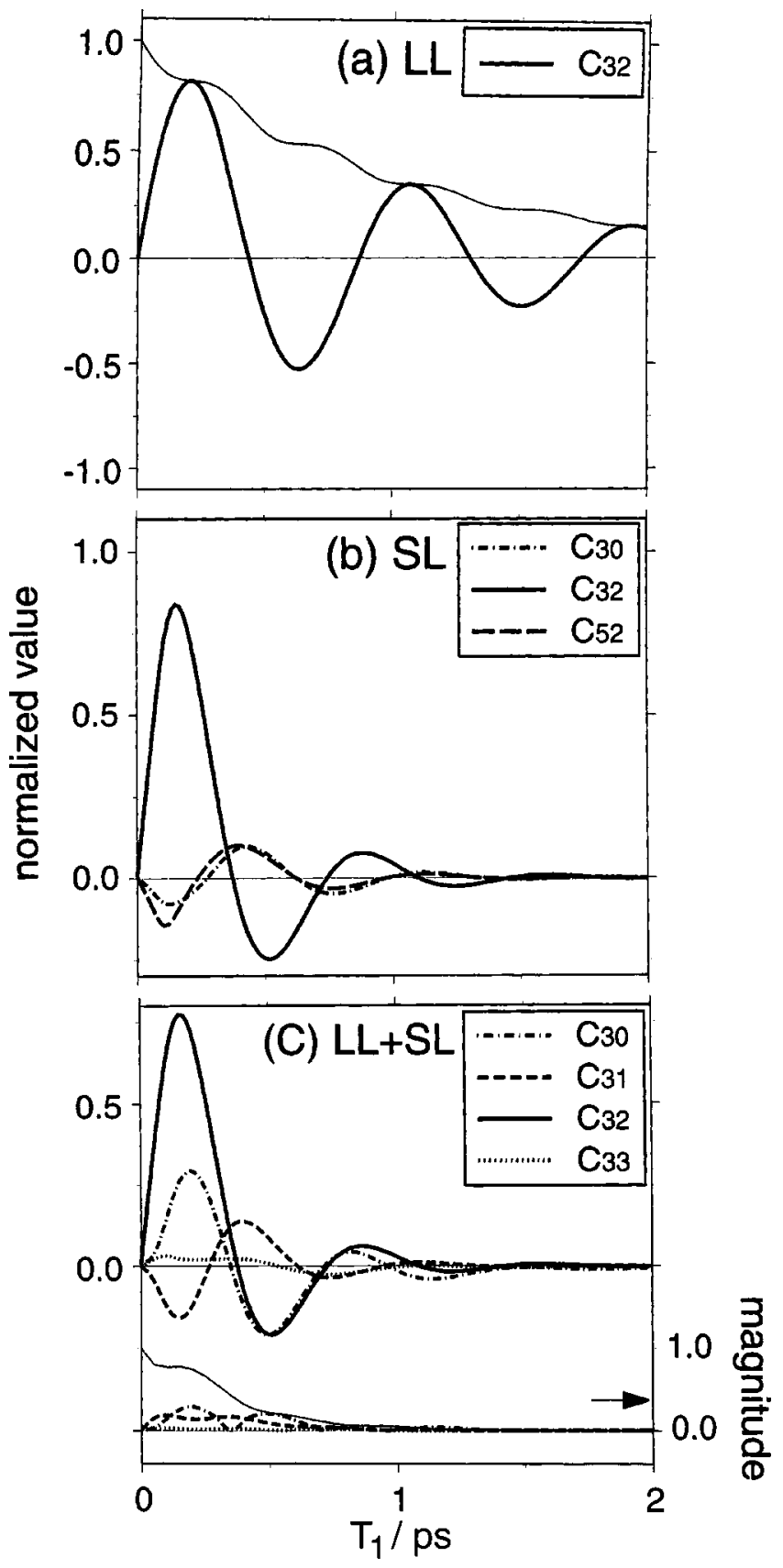

FIG. 5. The time evolution of the real part of $C_{30}$ (dotted-dashed line), $C_{31}$ (dashed line), $C_{32}$ (solid line), $C_{33}$ (dotted line), $C_{52}$ (long-dashed line) for the LL coupling (a), the SL coupling (b), and the LL plus SL coupling (c), respectively. Magnitudes of the matrix elements are normalized by $\left|C_{32}(0)\right|$. The absolute values are also plotted in panel (a) and the lower part of panel (c) (The thin solid line corresponds to $\left|C_{32}\left(T_{1}\right)\right| /\left|C_{32}(0)\right|$.)

case, and (c) the LL plus SL coupling case. Parameters are the same as used for the wave packet calculations. Figure 5(a) shows the calculated time evolution of coherence of $(n, m)=(3,2)$ for the LL coupling case [notice $\left|C_{n, m}(0)\right|$ has the maximum value at $(n, m)=(3,2)$ for the parameters used here], where all the coherences $C_{n+1, n}(t)$ have the same oscillation frequency $\Omega_{0}$ regardless of the quantum number. We only observed the coherences between the adjacent vibrational levels $C_{n+1, n}$ as predicted by Eqs. (4.6). The modulation of the coherence amplitude at the frequency $2 \Omega_{0}$, which is apparent from Eq. (4.6), reflects the wave packet rotation. The dissipative mechanism in the LL coupling with the Gaussian-white noise bath is regarded as the friction proportional to the momentum of the oscillator [see Eqs. (2.13) and (4.12)]; thus, the energy relaxation rate of the oscillator becomes maximum when the wave packet passes the potential minimum twice within a period of rotation. Therefore, the coherence decay is accelerated twice during $2 \pi / \Omega_{0}$. Since the energy relaxation rate of the system is shown to be time independent within the RWA, ${ }^{34}$ the modulation might be described beyond the RWA within the Redfield or weak coupling theory.

For the SL coupling model, the time evolution of the coherences for $(n, m)=(3,2),(3,0)$, and $(5,2)$ are plotted in Fig. 5(b), where coherences $C_{n+1, n}(t)$ show damped oscillations. Their frequencies are not as clearly identified as in the LL coupling case and depend on the quantum number (not shown). A distinctive difference from the LL coupling case is that there are relaxation induced coherences between the vibrational levels $(n+1, n \pm 2 m)$ as shown for $C_{3,0}(t)$. The exclusion of coherences between vibrational levels $(n+1, n$ $\pm 2 m+1$ ) can be explained by the symmetry of the system; the SL coupling term has odd parity with respect to the coordinate transformation as mentioned before and the parity of the density matrix elements $\phi_{n+1}(q) \phi_{n}\left(q^{\prime}\right)$ and $\phi_{n+1}(q) \phi_{n \pm 2 m}\left(q^{\prime}\right)$ are also odd for that transformation, but that of $\phi_{n+1}(q) \phi_{n \pm 2 m+1}\left(q^{\prime}\right)$ is even. Therefore, the even parity coherences $C_{n+1, n \pm 2 m+1}(t)$ cannot be created from the initial, odd-parity coherences $C_{n+1, n}(0)$ during the vibrational relaxation. We note that the specification of relaxation pathways might be complicated if we use the Redfield theory since the connection between the coherences $C_{n+1, n}$ and $C_{n+1, n-2}$, for example, cannot be treated within the RWA. ${ }^{34,35-37}$

Since the LL plus SL coupling terms do not possess the symmetry with respect to coordinate transformation, we can expect that all the coherences are created from the initial one-quantum coherences $C_{n+1, n}(0)$ for the LL plus SL coupling mechanism. Actually, this is the case shown in Fig. 5(c) for $n=2$, where all the coherences have nonzero values for $t>0$. Notice that only the coherence $C_{n+1, n}(t)$ is nonzero and the others are zero at $T_{1}=0 \mathrm{ps}$, since the latter are created through the vibrational relaxation processes. Only $C_{n+1, n \pm 2 m}(t)$ coherences are created even for the LL plus SL coupling when there is no overlap between the bath modes for the LL coupling and that for the SL coupling, in other words, when these two coupling mechanisms are statistically independent. Numerical calculations are done by setting cross terms $C_{1} C_{2}$ in Eqs. (2.11) to zero as mentioned in Sec. II B.

\section{B. Optical response functions}

In the previous subsection, we showed that the types of vibrational coherences created after the initial formation of the one-quantum coherences depend on the system-bath coupling mechanisms. In this subsection, we shall examine the temperature and the bath correlation time dependencies of the vibrational relaxation processes through the response functions. 
It is worth noting that since we assume a linear dependence of the polarizability (dipole) on the coordinate, the Raman (IR) response function [Eq. (3.4)] is proportional to a summation of the real part of the vibrational coherences

$$
R\left(T_{1}\right) \propto \sum_{n=0} \sqrt{n+1} \operatorname{Re}\left[C_{n+1, n}\left(T_{1}\right)\right] .
$$

This relation suggests that the response functions within the SL coupling model and the LL plus SL coupling model are similar, because the creation of $C_{n+1, n \pm 2 m}$ coherences are not detected directly by this order of vibrational spectroscopy. Since the SL coupling model was already discussed in the previous studies, ${ }^{15,16}$ we focus on the LL plus SL coupling model and discuss a few details, which have not been studied in the previous papers.

First, we consider the temperature dependence of the Raman response function. In Fig. 6(a), we plot the response function for the LL plus SL coupling at 150, 300, and $450 \mathrm{~K}$, and for comparison the response function for the LL coupling model. The other parameters are the same as in the previous subsection. The response function for the LL model does not show a temperature dependence, ${ }^{13}$ whereas that for the LL plus SL coupling model is temperature dependent such that the oscillation amplitude decreases with increasing temperature as shown in Fig. 6(a). When we turn to the pure SL coupling model $\left[C_{1} \equiv 0\right]$ with the same coupling parameter $\zeta_{S L}^{\prime}$, the patterns of the signal are almost identical with those for the LL plus SL coupling. The observed temperature dependence can be explained with the aid of the stochastic theory. ${ }^{30}$ If we regard the SL coupling term $-\hat{q}^{2} \sum_{j} g_{j} \hat{x}_{j} / 2$ [Eqs. (2.2) and (2.3)] as a part of the system potential, the deviation of the frequency from the zeroth order frequency $\omega_{0}$ can be denoted by $\delta \omega=X /\left(M \omega_{0}\right)$, where $X$ is the classical interaction coordinate corresponding to the quantum counterpart defined by Eq. (2.6). From Eq. (2.7) we have

$$
\langle\delta \omega(t) \delta \omega(0)\rangle_{B} \sim \frac{\zeta_{\mathrm{SL}} \gamma}{M \omega_{0}^{2} \beta} e^{-\gamma t} .
$$

This relation implies that the pure dephasing rate constant $\Gamma^{*}$ for the coherences $C_{n+1, n}(t)$ is $\zeta_{\mathrm{SL}} /\left(M \omega_{0}^{2} \beta\right)$ in the Gaussian-white limit $\gamma \rightarrow \infty$ (fast modulation limit), ${ }^{30}$ which is consistent with Eq. (2.16b) with $n-m=1$. Thus, the signal decays faster with increasing temperature and coupling strength. When the temperature becomes higher (or the coupling strength $\zeta_{\mathrm{SL}}^{\prime}$ becomes larger, not shown) the signal decays without changing sign, as can be seen in the plot for $T=450 \mathrm{~K}$. The origin of such behavior can be clarified by calculating the spectrum defined as

$$
R\left(\Omega_{1}\right)=\int_{0}^{\infty} d T_{1} e^{i \Omega_{1} T_{1}} R\left(T_{1}\right) .
$$

In Fig. 6(b), we plot the imaginary part of the spectrum $\operatorname{Im}\left[R\left(\Omega_{1}\right)\right]$. Note that this corresponds to the absorption spectrum in the resonant IR spectroscopy. We can see that the importance of the spectral contribution from the lower frequency region, which was attributed to energy relaxation through the $\hat{a}^{2}$ and $\hat{a}^{\dagger 2}$ terms included in the SL coupling, ${ }^{15}$ is increased at higher temperatures relative to that from the fundamental frequency region. Therefore, the oscillatory but constant sign decay pattern can be understood as a superposition of the positive background response from the broad lower frequency region and the oscillatory response from the fundamental frequency region.

Second, we investigate the effects of the correlation time $\tau_{c}=\gamma^{-1}$ on the spectral peak shift. Relations between the correlation time and the spectral shape are discussed in the previous papers. ${ }^{15,16}$ It is helpful to consider the classical Langevin equation corresponding to the Hamiltonian of Eq. $(2.1)^{7}$

$M \frac{d^{2} q(t)}{d t^{2}}+\frac{\partial U(q(t))}{\partial q(t)}+M \int_{0}^{\infty} d t^{\prime} \eta\left(t-t^{\prime}\right) \frac{d q\left(t^{\prime}\right)}{d t^{\prime}}=R(t)$,

where the friction kernel $\eta(t)$ and the random force $R(t)$ are related by a relation $\left\langle R(t) R\left(t^{\prime}\right)\right\rangle_{0}=M \eta\left(t-t^{\prime}\right) / \beta$, in which
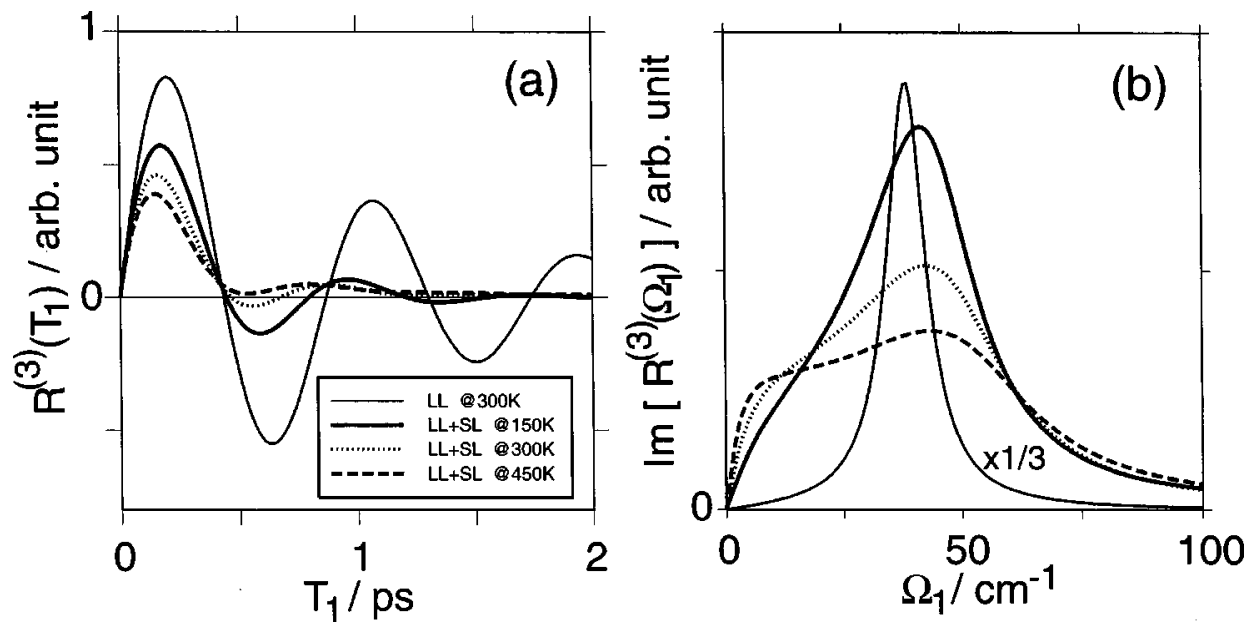

FIG. 6. Temperature dependence of the Raman response function (a) and its Fourier transform (b). The Raman response function for the LL coupling at 300 $\mathrm{K}$ (thin solid line, reduced by a factor 1/3), and the Raman response for the LL plus SL coupling at 150 (solid line), 300 (dotted line), and $450 \mathrm{~K}$ (dashed line). The other parameters are the same as used in Fig. 4. 


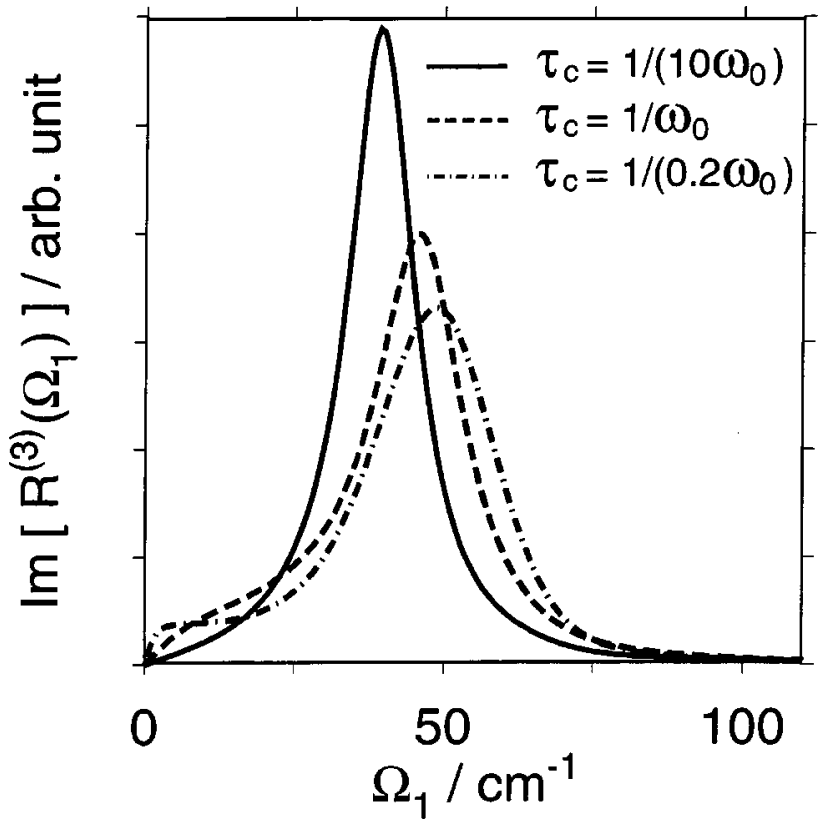

FIG. 7. The bath correlation time $\tau_{c}$ dependence of the Fourier transform of the Raman response function for the LL plus SL coupling model. The effective coupling strength $\zeta_{\mathrm{SL}}^{\prime} \gamma=0.05 \omega_{0}$ is fixed. The solid, dashed, and dotted-dashed lines represent, respectively, the response for $\left(\tau_{c} \omega_{0}, \zeta_{\mathrm{SL}}^{\prime}\right)$ $=(0.1,0.005),(1.0,0.05)$, and $(5.0,0.25)$. The other parameters are the same as used in Fig. 4.

$\langle\cdots\rangle_{0}$ denotes classical phase-space average with the initial distribution $e^{-\beta H}$. The friction kernel is expressed as

$$
\eta\left(t-t^{\prime}\right)=\frac{\partial V(q(t))}{\partial q(t)} \int d \omega \frac{J(\omega)}{M \omega} \cos \left\{\omega_{j}\left(t-t^{\prime}\right)\right\} \frac{\partial V\left(q\left(t^{\prime}\right)\right)}{\partial q\left(t^{\prime}\right)},
$$

where $V(q)$ is given by Eq. (2.3). For simplicity, we consider the SL coupling case $\left[C_{1} \equiv 0, C_{2} \equiv 1\right]$. For the GaussianMarkovian noise bath, substitution of Eq. (2.4) into Eq. (4.11) yields the friction part as

$$
\begin{gathered}
M \int_{0}^{\infty} d t^{\prime} \eta\left(t-t^{\prime}\right) \frac{d q\left(t^{\prime}\right)}{d t^{\prime}} \\
=M \zeta_{\mathrm{SL}} \gamma q(t)\left[q^{2}(t)-e^{-t / \tau_{c}} q^{2}(0)\right. \\
\left.\quad-\frac{1}{\tau_{c}} \int_{0}^{t} d \tau e^{-(t-\tau) / \tau_{c}} q^{2}(\tau)\right] .
\end{gathered}
$$

It should be noticed that the potential renormalization $\Delta U(\hat{q})=\Sigma_{j}\left[F_{j}(\hat{q})\right]^{2} /\left(2 m_{j} \omega_{j}^{2}\right)$ mentioned in Appendix A has already been eliminated in Eq. (4.10). ${ }^{7}$ Thus, the first term on the right hand side of Eq. (4.12) can contribute as an additional system anharmonic potential $M \zeta_{\mathrm{SL}} \gamma q^{4} / 4$ to the zeroth order potential $U(q)$ when the correlation time $\tau_{c}$ becomes long. The energy differences between any adjacent vibrational levels are always increased by such a potential within a first-order perturbation calculation. Therefore, we can expect that the spectral peak shift to the blue side is enhanced when the correlation time is long; however, its magnitude is inversely proportional to the correlation time.
This is the reason why the spectral peak has its highest value when $\tau_{c}$ is the order of $\omega_{0}^{-1}$, rather than $\tau_{c}=0$ (fast modulation) or $\tau_{c} \omega_{0} \gg 1$ (slow modulation) in Fig. 3 of Ref. 16. Note that the correlation of noise also causes an additional peak even in the LL case in the spectrum, if the bath is overdamped..$^{50,51}$

The additional anharmonic potential is proportional to $\gamma=1 / \tau_{c}$ and is related to the strength of the bath fluctuations by Eq. (2.7). Thus, to see the effect of the correlation time separately from the coupling strength, it is feasible to compare the responses for different $\tau_{c}$, but with a fixed effective coupling strength $\zeta_{\mathrm{SL}}^{\prime} \gamma$. In Fig. 7, we plot the imaginary part of the spectrum for the LL plus SL coupling model with $\zeta_{\mathrm{SL}}^{\prime} \gamma=0.05 \omega_{0}$ by varying the correlation time $\tau_{c}$. The solid, dashed, and dotted-dashed lines represent, respectively, the response for $\left(\tau_{c} \omega_{0}, \zeta_{\mathrm{SL}}^{\prime}\right)=(0.1,0.005),(1.0,0.05)$, and $(5.0,0.25)$. The other parameters are the same as used in Fig. 4. We can see that the gradual peak shifts to the blue side with increasing correlation time, as expected. Further, we clearly see that the spectral shape changes from a Lorentzian to a Gaussian-type shape, and its width becomes broader for longer correlation time as discussed in Ref. 16.

\section{CONCLUSION}

In this paper, we have derived the Fokker-Planck equation for an oscillator system in contact with a harmonic oscillator bath via linear-linear and square-linear coupling mechanisms. From this approach we are able to study the vibrational relaxation process under the simultaneous presence of elastic and inelastic interactions with the thermal bath. As an example of vibrational relaxation processes following photoexcitation, we investigated the vibrational response of a model harmonic oscillator system. From our model study, it is found that the conventional Brownian harmonic oscillator model (LL coupling model) and the pure SL coupling model considered in Refs. 14-16 induce very specific vibrational relaxation, i.e., in the former model, the only vibrational coherences between adjacent vibrational levels $C_{n+1, n}$, being created by the interaction with the pump laser pulse, are involved during the vibrational relaxation, whereas, in the later model, the coherences expressed as $C_{n+1, n \pm 2 m}$ are involved in addition to the initially created coherences. These selections of the relaxation pathways originate from the symmetry of the system-bath coupling. Only when the LL plus SL coupling mechanism is applied, in other words, one- and two-quantum transitions in the system oscillator take place simultaneously, are all the coherences generated during the vibrational relaxation.

Although the distinct differences of the vibrational relaxation pathways between the SL and the LL plus SL coupling model are not pronounced in the third-order Raman or linear IR response, we expect that such differences will be enhanced in higher-order vibrational spectroscopies. ${ }^{6,19}$ Since higher-order spectroscopy, such as fifth-order Raman and third-order IR spectroscopy, measures the vibrational relaxation process with more than two successive pumping processes, we are able to disentangle the several relaxation pathways involved, which allows us to investigate the nature of 
the system-bath interactions in more detail. ${ }^{19}$ Comparisons of the higher-order response functions with various systembath coupling conditions can be made by extending the present formulation, and will be carried out elsewhere.

Finally, applications of the present model should not be limited to vibrational spectroscopy; applications to similar problem such as chemical reaction ${ }^{17,18}$ and electronic spectroscopy ${ }^{52}$ provide us with further understanding of how dissipation plays a role in dynamics and are left for future studies.

\section{ACKNOWLEDGMENT}

One of the authors (Y.T.) thanks financial support of a Grant-in-Aid for Scientific Research (B) (12440171) from the Japan Society for the Promotion of Science.

\section{APPENDIX A: DERIVATION OF THE QUANTUM FOKKER-PLANCK EQUATION FOR THE GAUSSIAN-MARKOVIAN NOISE BATH}

In this Appendix, we derive the equation of motion for the reduced density matrix for the Gaussian-Markovian noise bath shown in Eq. (2.9). The derivation is similar to the LL coupling case (Ref. 17) and SL coupling case (Refs. 15 and 16), but here we take into account both interactions (LL + SL) simultaneously.

The dynamics of the system and the bath oscillators can be described by the density matrix $\rho_{\text {tot }}\left(q, \mathbf{x}, q^{\prime}, \mathbf{x}^{\prime}, t\right)$ $=\left\langle q, \mathbf{x}\left|\hat{\rho}_{\text {tot }}(t)\right| q^{\prime}, \mathbf{x}^{\prime}\right\rangle$ in the coordinate representation, where $\hat{\rho}_{\text {tot }}(t)$ is the density operator of the total system at time $t$, and $\mathbf{x}$ stands for all the coordinates of the bath modes. The optical response of the total system, however, can be calculated through the reduced density matrix elements defined by

$$
\rho\left(q, q^{\prime}, t\right) \equiv \int d \mathbf{x} \rho_{t o t}\left(q, \mathbf{x}, q^{\prime}, \mathbf{x}, t\right)
$$

since only the system variables are assumed to interact with the external electric field.

The reduced density matrix of the relevant system at time $t$ embedded in the harmonic oscillator bath at an inverse temperature $\beta$ in the coordinate representation is given by a functional integral form with an initial condition $\rho\left(q_{a}, q_{q}^{\prime}, t_{i}\right)$ as

$$
\begin{aligned}
\rho\left(q_{b}, q_{b}^{\prime}, t\right)= & \int_{-\infty}^{\infty} d q_{a} \int_{-\infty}^{\infty} d q_{a}^{\prime} \int_{q\left(t_{i}\right)=q_{a}}^{q(t)=q_{b}} \mathcal{D}[q] \\
& \times \int_{q^{\prime}\left(t_{i}\right)=q_{a}^{\prime}}^{q^{\prime}(t)=q_{b}^{\prime}} \mathcal{D}\left[q^{\prime}\right] \exp \left\{\frac{i}{\hbar} S[q]\right\} \\
& \times \exp \left\{-\frac{1}{\hbar} W\left[q, q^{\prime}\right]\right\} \\
& \times \exp \left\{-\frac{i}{\hbar} S\left[q^{\prime}\right]\right\} \rho\left(q_{a}, q_{a}^{\prime}, t_{i}\right),
\end{aligned}
$$

where the action $S$ is defined as

$$
S[q]=\int_{t_{i}}^{t} d \tau\left\{\frac{1}{2} M\left(\frac{d q(\tau)}{d \tau}\right)^{2}-U(q(\tau))\right\},
$$

and the exponent of the influence functional is explicitly given by ${ }^{31,53}$

$$
\begin{aligned}
W\left[q, q^{\prime}\right]= & \sum_{j} \frac{1}{2 m_{j} \omega_{j}} \int_{t_{i}}^{t} d \tau_{1} \int_{t_{i}}^{\tau_{1}} d \tau_{2}\left[F_{j}\left(q\left(\tau_{1}\right)\right)-F_{j}\left(q^{\prime}\left(\tau_{1}\right)\right)\right]\left\{\left[F_{j}\left(q\left(\tau_{2}\right)\right)-F_{j}\left(q^{\prime}\left(\tau_{2}\right)\right)\right] \operatorname{coth}\left(\beta \hbar \omega_{j} / 2\right)\right. \\
& \left.\times \cos \left[\omega_{j}\left(\tau_{1}-\tau_{2}\right)\right]-i\left[F_{j}\left(q\left(\tau_{2}\right)\right)+F_{j}\left(q^{\prime}\left(\tau_{2}\right)\right)\right] \sin \left[\omega_{j}\left(\tau_{1}-\tau_{2}\right)\right]\right\}+i \int_{t_{i}}^{t} d \tau\left[\Delta U(q(\tau))-\Delta U\left(q^{\prime}(\tau)\right)\right] .
\end{aligned}
$$

Note that the counter term $\Delta U(\hat{q})=\Sigma_{j}\left[F_{j}(\hat{q})\right]^{2} /\left(2 m_{j} \omega_{j}^{2}\right)$ found in Eq. (2.1) is taken into account as the second part on the right-hand side of Eq. (A3b) to cancel the potential renormalization of the system, which arises from the first part on the right-hand side of Eq. (A3b). The terms proportional to $\operatorname{coth}\left(\beta \hbar \omega_{j} / 2\right) \cos \left(\omega_{j} \tau\right)$ and $\sin \left(\omega_{j} \tau\right)$ on the right-hand side of Eq. (A3b) correspond to the real part and the imaginary part of the bath correlation function $C(t)=\langle\hat{X}(t) \hat{X}(0)\rangle_{B}$, respectively.

Within the high-temperature approximation, $\operatorname{coth}(\beta \hbar \gamma)$ $\simeq 1 /(\beta \hbar \gamma)$, we rewrite Eq. (A3b) by using Eqs. (2.3) and (2.4) as

$$
\begin{aligned}
\frac{1}{\hbar} W\left[q, q^{\prime}\right]= & \int_{t_{i}}^{t} d \tau_{1} \Phi\left(\tau_{1}\right) e^{-\gamma \tau_{1}}\left[\int_{t_{i}}^{\tau_{1}} d \tau_{2} e^{\gamma \tau_{2} \Theta\left(\tau_{2}\right)}\right. \\
& \left.+\frac{M}{2} \zeta_{\mathrm{LL}} \gamma e^{\gamma t_{i}}\left\{V\left(q_{a}\right)+V\left(q_{a}^{\prime}\right)\right\}\right],
\end{aligned}
$$

where

$$
\Phi(\tau)=\frac{i}{\hbar}\left\{V(q(\tau))-V\left(q^{\prime}(\tau)\right)\right\}
$$

and

$$
\begin{aligned}
\Theta(\tau)= & M \zeta_{\text {SL }} \gamma\left[\frac{1}{2} \frac{\partial V(q(\tau))}{\partial q(\tau)} \frac{d q(\tau)}{d \tau}\right. \\
& +\frac{1}{2} \frac{\partial V\left(q^{\prime}(\tau)\right)}{\partial q^{\prime}(\tau)} \frac{d q^{\prime}(\tau)}{d \tau} \\
& \left.-\frac{i}{\hbar \beta}\left\{V(q(\tau))-V\left(q^{\prime}(\tau)\right)\right\}\right]
\end{aligned}
$$

The detailed-balance condition is preserved in a semiclassical way as $C^{\prime \prime}(t)=(\hbar \beta / 2) \partial C^{\prime}(t) / \partial t$ with the real part $C^{\prime}(t)=S(t)$ of Eq. (2.7). 
Here, we define the auxiliary operator $\hat{\rho}_{n}(t)$ by its matrix element ${ }^{17}$ as

$$
\begin{aligned}
& \rho_{n}\left(q_{b}, q_{b}^{\prime}, t\right) \\
& \equiv \int_{-\infty}^{\infty} d q_{a} \int_{-\infty}^{\infty} d q_{a}^{\prime} \int_{q\left(t_{i}\right)=q_{a}}^{q(t)=q_{b}} \mathcal{D}[q] \int_{q^{\prime}\left(t_{i}\right)=q_{a}^{\prime}}^{q^{\prime}(t)=q_{b}^{\prime}} \mathcal{D}\left[q^{\prime}\right] \\
& \times\left[e^{-\gamma t}\left\{\int_{t_{i}}^{t} d \tau e^{\gamma \tau} \Theta(\tau)+c_{0}\right\}\right]^{n} \exp \left\{\frac{i}{\hbar} S[q]\right\} \\
& \times \exp \left\{-\frac{1}{\hbar} W\left[q, q^{\prime}\right]\right\} \exp \left\{-\frac{i}{\hbar} S\left[q^{\prime}\right]\right\} \rho\left(q_{a}, q_{a}^{\prime}, t_{i}\right),
\end{aligned}
$$

for integer $n \geqslant 0$, where the reduced density matrix is given by $\rho\left(q_{b}, q_{b}^{\prime}, t\right)=\rho_{0}\left(q_{b}, q_{b}^{\prime}, t\right)$. By taking the time derivative of Eq. (A6), we obtain a chain of equations of motion of the matrix elements as follows:

$$
\begin{aligned}
\frac{\partial}{\partial t} \rho_{0}\left(q, q^{\prime}, t\right)= & -\frac{i}{\hbar} L\left(q, q^{\prime}\right) \rho_{0}\left(q, q^{\prime}, t\right) \\
& -\Phi\left(q, q^{\prime}\right) \rho_{1}\left(q, q^{\prime}, t\right), \\
\frac{\partial}{\partial t} \rho_{1}\left(q, q^{\prime}, t\right)= & -\left\{\frac{i}{\hbar} L\left(q, q^{\prime}\right)+\gamma\right\} \rho_{1}\left(q, q^{\prime}, t\right) \\
& -\Phi\left(q, q^{\prime}\right) \rho_{2}\left(q, q^{\prime}, t\right) \\
& +\Theta\left(q, q^{\prime}\right) \rho_{0}\left(q, q^{\prime}, t\right), \\
\frac{\partial}{\partial t} \rho_{n}\left(q, q^{\prime}, t\right)= & -\left\{\frac{i}{\hbar} L\left(q, q^{\prime}\right)+n \gamma\right\} \rho_{n}\left(q, q^{\prime}, t\right) \\
& -\Phi\left(q, q^{\prime}\right) \rho_{n+1}\left(q, q^{\prime}, t\right) \\
& +n \Theta\left(q, q^{\prime}\right) \rho_{n-1}\left(q, q^{\prime}, t\right),
\end{aligned}
$$

where

$$
L\left(q, q^{\prime}\right) \equiv-\frac{\hbar^{2}}{2 M}\left(\frac{\partial^{2}}{\partial q^{2}}-\frac{\partial^{2}}{\partial q^{\prime 2}}\right)+U(q)-U\left(q^{\prime}\right)
$$

is the deterministic system Liouvillian in the coordinate representation. Relaxation operators $\Phi\left(q, q^{\prime}\right)$ and $\Theta\left(q, q^{\prime}\right)$ are obtained by changing $q(t) \rightarrow q, \quad q^{\prime}(t) \rightarrow q^{\prime}, \quad d q(\tau) / d \tau$ $\rightarrow(\hbar / i M) \partial / \partial q$, and $d q^{\prime}(\tau) / d \tau \rightarrow-(\hbar / i M) \partial / \partial q^{\prime}$ in Eqs. (A5a) and (A5b), respectively.

By introducing an anchor equation defined below, we can safely terminate the infinite hierarchy of the equations of motion

$$
\begin{aligned}
\frac{\partial}{\partial t} \rho_{N}\left(q, q^{\prime}, t\right)= & -\left\{\frac{i}{\hbar} L\left(q, q^{\prime}\right)+N \gamma\right\} \rho_{N}\left(q, q^{\prime}, t\right) \\
& -\frac{1}{\gamma} \Phi\left(q, q^{\prime}\right) \Theta\left(q, q^{\prime}\right) \rho_{N}\left(q, q^{\prime}, t\right) \\
& +N \Theta\left(q, q^{\prime}\right) \rho_{N-1}\left(q, q^{\prime}, t\right),
\end{aligned}
$$

for $n=N$, because the effects of the Gaussian-Markovian noise bath can be treated as if it is the Gaussian-white noise bath for large $N \gamma .{ }^{17}$ The depth of the hierarchy $N$ should be $N \gamma \gg \omega_{c}$, where $\omega_{c}$ is the characteristic frequency of the system. Obtaining a closed set of equations, we are able to calculate its time evolution with appropriate initial conditions $\hat{\rho}_{n}(t=0)$ for $0 \leqslant n \leqslant N$. Wigner transformation of Eqs. (A7) and (A9) in accordance with the definition of Eq. (2.8) and the operator relations described in Appendix B results in the Fokker-Planck equation of Eqs. (2.9).

\section{APPENDIX B: RELATIONS BETWEEN HILBERT SPACE OPERATORS AND ITS WIGNER REPRESENTATIONS}

Here, we list the relations between Hilbert space operators and its Wigner representations following Ref. 54.

We make the Wigner transformation of the auxiliary density matrix elements yield the auxiliary distribution functions, ${ }^{32,54}$

$$
W_{n}(p, q, t) \equiv \frac{1}{2 \pi \hbar} \int d x e^{i p x / \hbar} \rho_{n}\left(q-\frac{x}{2}, q+\frac{x}{2}, t\right) .
$$

The corresponding Wigner operators are derived by rewriting the operators in Eq. (A7) in terms of $\hat{p} \equiv-i \hbar \partial / \partial q$ and $\hat{q}$, then transforming to the Wigner space expressions via the relations ${ }^{54}$

$$
\begin{aligned}
& A(\hat{p}, \hat{q}) \hat{\rho}_{n}(t) \rightarrow A\left(p+\frac{\hbar}{2 i} \frac{\partial}{\partial q}, q-\frac{\hbar}{2 i} \frac{\partial}{\partial p}\right) W_{n}(p, q, t), \\
& \hat{\rho}_{n}(t) A(\hat{p}, \hat{q}) \rightarrow A\left(p-\frac{\hbar}{2 i} \frac{\partial}{\partial q}, q+\frac{\hbar}{2 i} \frac{\partial}{\partial p}\right) W_{n}(p, q, t) .
\end{aligned}
$$

Integral expression relations for a commutator

$\frac{i}{\hbar}\{A(\hat{q}) \hat{\rho}-\hat{\rho} A(\hat{q})\} \rightarrow \int_{-\infty}^{\infty} d p^{\prime} A_{W}\left(p-p^{\prime}, q\right) W\left(p^{\prime}, q, t\right)$,

with

$A_{W}(p, q)=\frac{1}{\hbar} \int_{-\infty}^{\infty} \frac{d x}{2 \pi \hbar} \sin (p x / \hbar)\left\{A\left(q+\frac{x}{2}\right)-A\left(q-\frac{x}{2}\right)\right\}$,

and for an anticommutator

$$
\frac{1}{2}\{A(\hat{q}) \hat{\rho}+\hat{\rho} A(\hat{q})\} \rightarrow \int_{-\infty}^{\infty} d p^{\prime} A_{W}^{\circ}\left(p-p^{\prime}, q\right) W\left(p^{\prime}, q, t\right),
$$

with

$A_{W}{ }^{\circ}(p, q)=\frac{1}{2} \int_{-\infty}^{\infty} \frac{d x}{2 \pi \hbar} \cos (p x / \hbar)\left\{A\left(q+\frac{x}{2}\right)+A\left(q-\frac{x}{2}\right)\right\}$,

are also useful, and are derived from the relations

$$
\int_{-\infty}^{\infty} d x e^{-i p x / \hbar} \frac{\partial}{\partial x} F(q, x)=\frac{i}{\hbar} p \int_{-\infty}^{\infty} d r e^{-i p x / \hbar} F(q, x),
$$

and

$e^{-i p x / \hbar} U\left(q+\frac{x}{2}\right) F(q, x)=U\left(q-\frac{\hbar}{2 i} \frac{\partial}{\partial p}\right) e^{-i p x / \hbar} F(q, x)$. 


\section{APPENDIX C: ROTATING WAVE APPROXIMATION AND REDFIELD TENSOR ELEMENTS}

As was discussed in Ref. 52, the effective coupling strength between the system and the bath in the GaussianMarkovian hierarchy equations is inversely proportional to $\gamma$. Thus, the Gaussian-white equation, which is the white noise limit, i.e., $\gamma \rightarrow \infty$ for $\gamma \gg \omega_{c}$, where $\omega_{c}$ is the characteristic frequency of the system, of Gaussian-Markovian equations, reduces to the same form as one obtained from the second-order perturbation theory (see also Ref. 44).

In this Appendix, we rewrite the damping operator of the Gaussian-white Fokker-Planck equation [Eq. (2.14)] for the LL plus SL model and show that under the rotating wave approximation the damping operator yields similar (not exactly the same) Redfield tensor elements given by Eqs. (2.15a). The damping operator $\Gamma_{W}(p, q)$ in the FokkerPlanck equation [Eq. (2.11c)] is expressed in terms of $\hat{p}$ and $\hat{q}$ as

$$
\begin{aligned}
\hat{\Gamma} \hat{\rho}= & -\frac{i}{\hbar} \zeta[V(\hat{q}), F(\hat{q}) \hat{p} \hat{\rho}]-\frac{i}{\hbar} \zeta[V(\hat{q}), \hat{\rho} \hat{p} F(\hat{q})] \\
& -\frac{M \zeta}{\hbar^{2} \beta}[V(\hat{q}),[V(\hat{q}), \hat{\rho}]],
\end{aligned}
$$

where $\hat{\rho}$ is the reduced density matrix of the system and $F(\hat{q}) \equiv 1 / 2 \partial V(\hat{q}) / \partial \hat{q}$ with $V(\hat{q})$ given by Eq. $(2.3)$. We express the damping operator in the energy-level representation by using $\hat{p}=i \sqrt{M \hbar \omega_{0} / 2}\left(\hat{a}^{\dagger}-\hat{a}\right)$ and $\hat{q}=\sqrt{\hbar /\left(2 M \omega_{0}\right)}\left(\hat{a}^{\dagger}\right.$ $+\hat{a})$. We then split the operator into three parts as

$$
\hat{\Gamma} \equiv \hat{\Gamma}_{\text {RWA }}+\hat{\Gamma}_{\text {nonRWA }}+\hat{\Gamma}_{\text {cross }},
$$

where

$$
\begin{aligned}
\hat{\Gamma}_{\mathrm{RWA}} \hat{\rho}= & \frac{\zeta_{\mathrm{LL}}}{2}\left(Z^{(+)}\left(\omega_{0}\right)\left\{\left[\hat{a}, \hat{\rho} \hat{a}^{\dagger}\right]+\text { h.c. }\right\}+Z^{(-)}\left(\omega_{0}\right)\left\{\left[\hat{a} \hat{a}^{\dagger}, \hat{\rho} \hat{a}\right]+\text { h.c. }\right\}\right)+\frac{\hbar \zeta_{\mathrm{SL}}}{2 M \omega_{0}}\left(Z^{(+)}\left(2 \omega_{0}\right)\left\{\left[\hat{a}^{2}, \hat{\rho} \hat{a}^{\dagger 2}\right]+\text { h.c. }\right\}\right. \\
& \left.+Z^{(-)}\left(2 \omega_{0}\right)\left\{\left[\hat{a}^{\dagger 2}, \hat{\rho} \hat{a}^{2}\right]+\text { h.c. }\right\}\right)-\frac{\zeta_{\mathrm{SL}}}{\beta M \omega_{0}^{2}}[\hat{n},[\hat{n}, \hat{\rho}]], \\
\hat{\Gamma}_{\mathrm{nonRWA}} \hat{\rho}= & \frac{\zeta_{\mathrm{LL}}}{2}\left(Z^{(+)}\left(\omega_{0}\right)\left\{\left[\hat{a}^{\dagger}, \hat{\rho} \hat{a}^{\dagger}\right]+\text { h.c. }\right\}+Z^{(-)}\left(\omega_{0}\right)\{[\hat{a}, \hat{\rho} \hat{a}]+\text { h.c. }\}\right)+\frac{\hbar \zeta_{\mathrm{SL}}}{2 M \omega_{0}}\left(Z^{(+)}\left(2 \omega_{0}\right)\left\{\left[\hat{a}^{\dagger 2}, \hat{\rho} \hat{a}^{\dagger 2}\right]+\text { h.c. }\right\}\right. \\
& +Z^{(-)}\left(2 \omega_{0}\right)\left\{\left[\hat{a}^{2}, \hat{\rho} \hat{a}^{2}\right]+\text { h.c. }\right\}+Z^{(+)}\left(\omega_{0}\right)\left\{\left[2 \hat{n}, \hat{\rho} \hat{a}^{\dagger 2}\right]+\text { h.c. }\right\}+Z^{(-)}\left(\omega_{0}\right)\left\{[2 \hat{n}, \hat{\rho}] \hat{a}^{2}-2 \hat{\rho} \hat{a}^{2}+\text { h.c. }\right\} \\
& \left.-\left\{\frac{2}{\beta \hbar \omega_{0}} \hat{a}^{2} \hat{\rho}+\left(\hat{a}^{\dagger 2}-\hat{a}^{2}\right) \hat{\rho} \hat{n}+\text { h.c. }\right\}\right)
\end{aligned}
$$

and

$$
\begin{aligned}
\hat{\Gamma}_{\text {cross }} \hat{\rho}= & \zeta_{\text {cross }}\left(\left(Z^{(-)}\left(\omega_{0}\right)-1\right)\left\{\left[\hat{a}^{\dagger}, \hat{a}^{\dagger 2} \hat{\rho}\right]+\text { h.c. }\right\}+\left(Z^{(-)}\left(\omega_{0}\right)+2\right)\left\{\left[\hat{a}, \hat{a}^{2} \hat{\rho}\right]+\text { h.c. }\right\}+3 Z^{(-)}\left(\omega_{0}\right)\left\{\hat{a}^{\dagger}(\hat{n}+1) \hat{\rho}+\text { h.c. }\right\}\right. \\
& +3\left(Z^{(-)}\left(\omega_{0}\right)+1\right)\{(\hat{n}+1) \hat{a} \hat{\rho}+\text { h.c. }\}-2 Z^{(-)}\left(2 \omega_{0}\right)\left\{\hat{a} \dagger \hat{\rho} \hat{a}^{\dagger 2}+\text { h.c. }\right\}-2\left(Z^{(-)}\left(2 \omega_{0}\right)+1\right)\left\{\hat{a} \hat{\rho} \hat{a}^{2}+\text { h.c. }\right\} \\
& -\left(2 Z^{(-)}\left(2 \omega_{0}\right)+Z^{(-)}\left(\omega_{0}\right)\right)\left\{\hat{a} \hat{a}^{\dagger} \hat{\rho} \hat{a}^{2}+\text { h.c. }\right\}-\left(2 Z^{(-)}\left(2 \omega_{0}\right)+Z^{(-)}\left(\omega_{0}\right)+3\right)\left\{\hat{a} \hat{\rho} \hat{a}^{\dagger 2}+\text { h.c. }\right\} \\
& -\left(2 Z^{(-)}\left(2 \omega_{0}\right)+Z^{(-)}\left(\omega_{0}\right)+1\right)\left\{\hat{a}^{\dagger} \hat{\rho}\left(\hat{a}^{[6]} \hat{a}+\hat{a} \hat{a}^{\dagger}\right)+\text { h.c. }\right\}-\left(2 Z^{(-)}\left(2 \omega_{0}\right)+Z^{(-)}\left(\omega_{0}\right)+2\right)\left\{\hat{a} \hat{\rho}\left(\hat{a} \hat{a}^{\dagger} \hat{a}+\hat{a} \hat{a}^{\dagger}\right)+\text { h.c. }\right\} \\
& \left.-\left\{6 \hat{a}^{\dagger} \hat{\rho}+6 \hat{a} \hat{\rho}-\left(\hat{a}^{\dagger 2}+\hat{a}^{2}\right) \hat{\rho}+\text { h.c. }\right\} / 2\right) .
\end{aligned}
$$

Here, the system-bath coupling constants, $\zeta_{\mathrm{LL}}=4\left[C_{1}\right]^{2} \zeta$ and $\zeta_{\mathrm{SL}}=C_{2} \zeta$, are defined in the text and we used notations; $\zeta_{\text {cross }} \equiv-\zeta C_{1} C_{2} \sqrt{\hbar /\left(2 M \omega_{0}\right)}, Z^{( \pm)}\left(\omega_{0}\right) \equiv\left(1 \pm \beta \hbar \omega_{0} / 2\right) /\left(\beta \hbar \omega_{0}\right)$, and $\hat{n}=\hat{a}^{\dagger} \hat{a}$. Operator $\hat{\Gamma}_{\mathrm{RWA}}$ represents the contribution with the rotating wave approximation (RWA), whereas $\hat{\Gamma}_{\text {nonRWA }}$ and $\hat{\Gamma}_{\text {cross }}$ represent the remaining terms. Note that we included the cross terms of LL and SL interaction proportional to $C_{1} C_{2}$ to $\Gamma_{\text {cross }}$. The first and the second terms on the right-hand side of Eq. (C2b) represent the energy dissipation by one- and two-quantum transitions, respectively, and the third term represents the pure dephasing processes. The effects of the LL and the SL couplings are decoupled in $\Gamma_{\mathrm{RWA}}$ since cross terms are neglected within the rotating wave approximation. Note that we do not have the imaginary terms which shift the system frequency since we included the counter term in the Hamiltonian. The operator $\Gamma_{\mathrm{RWA}}$ is expressed in the tensor form as 


$$
\begin{aligned}
\left(\Gamma_{\mathrm{RWA}}\right)_{n, m ; r, s}= & \frac{\zeta_{\mathrm{LL}}}{2}\left[Z^{(+)}\left(\omega_{0}\right)\left\{2 \sqrt{r s} \delta_{r, n+1} \delta_{s, m+1}-(r+s) \delta_{r, n} \delta_{s, m}\right\}+Z^{(-)}\left(\omega_{0}\right)\left\{2 \sqrt{(r+1)(s+1)} \delta_{r, n-1} \delta_{s, m-1}\right.\right. \\
& \left.\left.-(r+s+2) \delta_{r, n} \delta_{s, m}\right\}\right]+\frac{\hbar \zeta_{\mathrm{SL}}}{2 M \omega_{0}}\left[Z ^ { ( + ) } ( 2 \omega _ { 0 } ) \left\{2 \sqrt{r(r-1) s(s-1)} \delta_{r, n+2} \delta_{s, m+2}\right.\right. \\
& \left.-(r(r-1)+s(s-1)) \delta_{r, n} \delta_{s, m}\right\}+Z^{(-)}\left(2 \omega_{0}\right)\left\{2 \sqrt{(r+1)(r+2)(s+1)(s+2)} \delta_{r, n-2} \delta_{s, m-2}\right. \\
& \left.\left.-((r+1)(r+2)+(s+1)(s+2)) \delta_{r, n} \delta_{s, m}\right\}\right]-\frac{\zeta_{\mathrm{SL}}}{\beta M \omega_{0}^{2}}(r-s)^{2} \delta_{r, n} \delta_{s, m} .
\end{aligned}
$$

Since $N\left(\omega_{0}\right) \sim Z^{(-)}\left(\omega_{0}\right)$ and $N\left(2 \omega_{0}\right) \sim Z^{(-)}\left(2 \omega_{0}\right)$ for the high-temperature condition, the above expression agree with the Redfield tensor elements given in Eqs. (2.16). Therefore, we clearly showed that under the rotating wave approximation the Gaussian-white Fokker-Planck equation reduces to the Redfield description in the RWA. We note, however, that the Redfield theory does not have a limitation for a temperature ${ }^{43}$ in contrast to the Fokker-Planck equation approach. One often uses the Redfield theory or the response function theory by adapting the energy-level representation to study molecular vibrational motion in condensed phase, but the situation in the vibrational energy states is, in many cases, different from the electronic states. The most natural expression of the vibrational motion is in the coordinate representation and one should go back to the original Hamiltonian, if applicability of the theory is not certain.

${ }^{1}$ M. Buchner, B. M. Ladanyi, and R. M. Stratt, J. Chem. Phys. 97, 8522 (1992).

${ }^{2}$ R. M. Stratt and M. Cho, J. Chem. Phys. 100, 6700 (1994).

${ }^{3}$ S. Saito and I. Ohmine, J. Chem. Phys. 106, 4889 (1997); 108, 240 (1998).

${ }^{4}$ R. L. Murry, J. T. Fourkas, and T. Keyes, J. Chem. Phys. 109, 2814 (1998); 109, 7913 (1998).

${ }^{5}$ T. Keyes, and J. T. Fourkas, J. Chem. Phys. 112, 287 (2000).

${ }^{6}$ S. Mukamel, Principles of Nonlinear Optical Spectroscopy (Oxford University Press, New York, 1995).

${ }^{7}$ U. Weiss, Quantum Dissipative Systems, 2nd ed. (World Scientific, Singapore, 1999).

${ }^{8}$ H. Grabert, P. Schramm, and G. L. Ingold, Phys. Rep. 168, 115 (1988).

${ }^{9}$ P. Hänggi, P. Talkner, and M. Borkovec, Rev. Mod. Phys. 62, 251 (1990).

${ }^{10}$ A. O. Caldeira and A.J. Leggett, Physica A 121, 587 (1993).

${ }^{11}$ Y. J. Yan and S. Mukamel, J. Chem. Phys. 89, 5160 (1988); 94, 179 (1991).

${ }^{12}$ Y. Tanimura and S. Mukamel, Phys. Rev. E 47, 118 (1993).

${ }^{13}$ K. Okumura and Y. Tanimura, Phys. Rev. E 53, 214 (1996).

${ }^{14}$ K. Okumura and Y. Tanimura, Phys. Rev. E 56, 2747 (1997).

${ }^{15}$ T. Steffen and Y. Tanimura, J. Phys. Soc. Jpn. 69, 3115 (2000).

${ }^{16}$ Y. Tanimura and T. Steffen, J. Phys. Soc. Jpn. 69, 4095 (2000).

${ }^{17}$ Y. Tanimura and P. G. Wolynes, Phys. Rev. A 43, 4131 (1991).

${ }^{18}$ Y. Tanimura and P. G. Wolynes, J. Chem. Phys. 96, 8485 (1992).

${ }^{19}$ Y. Tanimura and S. Mukamel, J. Chem. Phys. 99, 9496 (1993).
${ }^{20}$ T. Steffen J. T. Fourkas, and K. Duppen, J. Chem. Phys. 105, 7364 (1996).

${ }^{21}$ T. Steffen and K. Duppen, Chem. Phys. 233, 267 (1997).

${ }^{22}$ J. T. Fourkas, H. Kawashima, and K. A. Nelson, J. Chem. Phys. 103, 4393 (1995).

${ }^{23}$ T. Mikami, M. Shiga, and S. Okazaki, J. Chem. Phys. 115, 9797 (2001).

${ }^{24}$ A. Garg, J. N. Onuchic, and V. Ambergaokar, J. Chem. Phys. 83, 4491 (1985).

${ }^{25}$ Y. Tanimura and S. Mukamel, J. Chem. Phys. 101, 3049 (1994).

${ }^{26}$ D. W. Oxtoby, Adv. Chem. Phys. 40, 1 (1979); 47, 487 (1981).

${ }^{27}$ J. S. Bader and B. J. Berne, J. Chem. Phys. 100, 8359 (1994).

${ }^{28}$ W. T. Pollard and R. A. Friesner, J. Chem. Phys. 100, 5054 (1994).

${ }^{29}$ Y. Tanimura and R. Kubo, J. Phys. Soc. Jpn. 68, 101 (1989).

${ }^{30}$ R. Kubo, M. Toda, and N. Hashitsume, Statistical Mechanics (Springer, New York, 1985).

${ }^{31}$ R. P. Feynman and F. L. Vernon, Ann. Phys. (N.Y.) 24, 118 (1963).

${ }^{32}$ E. Wigner, Phys. Rev. 40, 749 (1932).

${ }^{33}$ I. Kondov, U. Kleinekathöfer, and M. Schrieiber, J. Chem. Phys. 114, 1497 (2001).

${ }^{34}$ D. Kohen, C. C. Marston, and D. J. Tannor, J. Phys. Chem. A 107, 5236 (1997).

${ }^{35}$ A. G. Redfield, Adv. Magn. Reson. 1, 1 (1965).

${ }^{36}$ K. Blum, Density Matrix Theory and Applications (Plenum, New York, 1981).

${ }^{37}$ W. T. Pollard, A. K. Felts, and R. A. Friesner, Adv. Chem. Phys. 93, 77 (1996).

${ }^{38}$ P. A. Madden and R. M. Lyden-Bell, Chem. Phys. Lett. 38, 163 (1976).

${ }^{39}$ D. W. Oxtoby and S. A. Rice, Chem. Phys. Lett. 42, 1 (1976).

${ }^{40}$ A. B. Myers and F. Markel, Chem. Phys. 149, 21 (1990).

${ }^{41}$ T. Yamaguchi, J. Chem. Phys. 112, 8530 (2000).

${ }^{42}$ Y. J. Yan, Phys. Rev. A 58, 2721 (1998).

${ }^{43}$ Y. J. Yan, F. Shuang, R. Xu, J. Cheng, X. Q. Li, C. Yang, and H. Zhang, J. Chem. Phys. 113, 2068 (2000).

${ }^{44}$ J. Cao, J. Chem. Phys. 107, 3204 (1997)

${ }^{45}$ S. Jang, J. Cao, and R. Silbey, J. Chem. Phys. 116, 2705 (2002).

${ }^{46}$ Y. Tanimura, Chem. Phys. 233, 217 (1998).

${ }^{47}$ R. Karrlein and H. Grabert, Phys. Rev. E 55, 153 (1997).

${ }^{48}$ C. Meier and D. J. Tannor, J. Chem. Phys. 111, 3365 (1999).

${ }^{49}$ W. R. Frensley, Rev. Mod. Phys. 62, 745 (1990).

${ }^{50}$ Y. Suzuki and Y. Tanimura, Chem. Phys. Lett. (in press).

${ }^{51}$ Y. Suzuki and Y. Tanimura, J. Phys. Soc. Jpn. (submitted).

${ }^{52}$ Y. Tanimura and Y. Maruyama, J. Chem. Phys. 107, 1779 (1997); Y. Maruyama and Y. Tanimura, Chem. Phys. Lett. 292, 28 (1998).

${ }^{53}$ R. P. Feynman and A. R. Hibbs, Quantum Mechanics and Path Integrals (McGraw-Hill, New York, 1965).

${ }^{54}$ R. Kubo, J. Phys. Soc. Jpn. 19, 2127 (1964). 